\title{
HYPERARITHMETICAL INDEX SETS IN RECURSION THEORY
}

\author{
STEFFEN LEMPP
}

\begin{abstract}
We define a family of properties on hyperhypersimple sets and show that they yield index sets at each level of the hyperarithmetical hierarchy. An extension yields a $\Pi_{1}^{1}$-complete index set. We also classify the index set of quasimaximal sets, of coinfinite r.e. sets not having an atomless superset, and of r.e. sets major in a fixed nonrecursive r.e. set.
\end{abstract}

0. Introduction. The present paper deals with index sets, i.e., sets of indices of partial recursive (p.r.) functions and recursively enumerable (r.e.) sets that are defined through the p.r. functions or r.e. sets they code. The early results in index sets used geometric arguments in one- or two-dimensional arrays: Rogers showed the $\Sigma_{3}$ and $\Pi_{3}$-completeness of the index sets of recursive and simple sets, respectively, in a finite injury argument. Lachlan, D. A. Martin, R. W. Robinson, and Yates (1968, unpublished, later appearing in Tulloss [Tu71]) showed the $\Pi_{4}$-completeness of the index set of maximal sets in an infinite injury argument. Tulloss [ibid.] also mentions for the first time the question whether the index set of quasimaximal sets is $\Sigma_{5}$-complete. However, the geometric method was too complex at higher levels of the arithmetical hierarchy. During the 1970's, progress in index sets was mainly made in other areas by several Russian mathematicians as well as L. Hay.

Schwarz [Schta] was the first to introduce induction into index set proofs (in the r.e. degrees) and was able to show that the index sets of $\operatorname{low}_{n}$ and high $\mathrm{h}_{n}$ r.e. sets are $\Sigma_{n+3}$ and $\Sigma_{n+4}$-complete, respectively. Solovay [JLSSta] then extended Schwarz's methods to show the $\Sigma_{\omega+1}$-completeness of the index sets of low $<\omega$ (low $n$ for some $n$ ) and of high $<\omega\left(\right.$ high $_{n}$ for some $\left.n\right)$ r.e. sets as well as the $\Pi_{\omega+1}$-completeness of the index set of intermediate degrees (degrees neither low $<\omega$ nor high $<\omega$ ).

In this paper, we exhibit a family of algebraically invariant properties $\mathbf{L}_{\omega_{1}, \omega^{-}}$ definable in $\mathcal{E}$, that yields index sets at any level of the hyperarithmetical hierarchy. The proof is based on induction and Lachlan's theorem [La68] that any $\Sigma_{3}$-Boolean algebra is isomorphic to the lattice of r.e. supersets of some r.e. set (modulo finite sets). It uses tree arguments and the fact that the Cantor-Bendixson rank of a tree corresponds to certain properties of the lattice of r.e. supersets of the set constructed. An extension yields a $\Pi_{1}^{1}$-complete index set. A corollary shows the $\Sigma_{5}$-completeness of the index set of quasimaximal sets, thereby settling this longopen question. Further results classify the index sets of atomic sets and of r.e. sets major in a fixed nonrecursive r.e. set.

Our notation is fairly standard and generally follows Soare's forthcoming book Recursively Enumerable Sets and Degrees [Sota].

Received by the editors July 7, 1986 and, in revised form, November 5, 1986.

1980 Mathematics Subject Classification (1985 Revision). Primary 03D25.

This paper is an extended version of part of the author's thesis. He wishes to thank his thesis advisor, R. I. Soare, as well as T. A. Slaman and J. Steel for helpful suggestions and comments. 
We consider sets and functions on the natural numbers $\omega=\{0,1,2,3, \ldots\}$. For a partial function $\varphi, \varphi(x) \downarrow$ denotes that $x \in \operatorname{dom} \varphi$, otherwise we write $\varphi(x) \uparrow$. We identify a set $A$ with its characteristic function $\chi_{A} . f \uparrow x$ denotes $f$ restricted to arguments less than $x$, likewise for sets.

We let $A \subset B$ denote that $A \subseteq B$ but $A \neq B ; A \subseteq^{*} B$ that $A-B$ is finite; and $A \subset_{\infty} B$ that $A \subseteq B$ and $|B-A|=\infty$. $\bar{A}$ will denote the complement of $A$, $A \sqcup B$ the disjoint union. For each $n \in \omega$, we let $\left\langle x_{1}, x_{2}, \ldots, x_{n}\right\rangle$ denote the coded $n$-tuple (where $x_{i} \leq\left\langle x_{1}, x_{2}, \ldots, x_{n}\right\rangle$ for each $i$ ).

In a partial order, $x \mid y$ denotes that $x$ and $y$ are incomparable. $[k, l)$ denotes the interval $\{n \in \omega \mid k \leq n<l\}$.

The logical connectives "and" and "or" will be denoted by $\wedge$ and $\vee$, respectively. We allow as additional quantifiers (in the meta-language) $\left(\exists^{\infty} x\right),\left(\exists^{<\infty} x\right)$, and (a.e. $x$ ) to denote that the set of such $x$ is infinite, finite, and cofinite, respectively.

$\{e\}$ (or $\varphi_{e}$ ) and $W_{e}\left(\{e\}^{X}\right.$ (or $\Phi_{e}^{X}$ ) and $W_{e}^{X}$ ) denote the eth partial recursive function and its domain (with oracle $X$ ) under some fixed standard numbering. $\leq_{1}$ and $\leq_{T}$ denote one-one and Turing reducibility, respectively, and $\equiv_{1}$ and $\equiv_{T}$ the induced equivalence relations.

In the context of trees, $\rho, \sigma, \tau, \ldots$ denote finite strings; $|\sigma|$ the length of $\sigma ; \sigma^{\wedge} \tau$ the concatenation of $\sigma$ and $\tau ;\langle a\rangle$ the one-element string consisting of $a ;\left\langle a^{n} b^{m} \ldots\right\rangle$ the finite string consisting of $n$ many $a$ 's, followed by $m$ many $b$ 's, . ; $\sigma \subseteq \tau(\sigma \subset \tau)$ that $\sigma$ is a (proper) initial segment of $\tau ; \sigma<_{L} \tau$ that for some $i, \sigma \uparrow i=\tau \uparrow i$ and $\sigma(i)<_{\Lambda} \tau(i)$ (where $<_{\Lambda}$ is a given order on $\Lambda$ and $T \subseteq \Lambda^{<\omega}$ ); and $\sigma \leq \tau(\sigma<\tau)$ that $\sigma<_{L} \tau$ or $\sigma \subseteq \tau(\sigma \subset \tau)$.

The set $[T]$ of infinite paths through a tree $T \subseteq \Lambda^{<\omega}$ is $\left\{p \in \Lambda^{\omega} \mid(\forall n)[p \uparrow n \in\right.$ $T]\}$. The extendible part of a tree $T$ is $\{\sigma \in T \mid(\exists p \in[T])[\sigma \subset p]\}$. The part of a tree above $\sigma$ is $T(\sigma)=\left\{\tau \mid \sigma^{\wedge} \tau \in T\right\}$.

We will first prove an easy warm-up theorem to demonstrate our technique for index set classifications in a simple setting. It reproves previously known results and classifies for the first time the index sets of quasimaximal sets and of coinfinite r.e. sets not having atomless supersets (the so-called atomic sets) as $\Sigma_{5^{-}}$and $\Pi_{6}$ complete, respectively.

First of all, however, we will explain the tree machinery needed to prove the main results of this chapter. All trees using this machinery will from now on be binary.

1. The machinery. Lachlan [La68] showed that any $\Sigma_{3}$-Boolean algebra can be represented as the lattice of r.e. supersets (modulo finite sets) of some hyperhypersimple set $A$. The proof uses an argument that can be generalized substantially. From an arbitrary $\Sigma_{2}$-tree $T \in 2^{<\omega}$ (i.e., $\sigma \in T$ iff $R(\sigma)$, for some $\Sigma_{2}$-predicate $R$ ), Lachlan constructs a (hyperhypersimple) r.e. set $A_{T}$ with a 1-1 correspondence between nodes $\sigma \in T$ and elements $a_{\sigma} \in \bar{A}$ satisfying the following two properties:

(i) $(\forall \sigma \in T)\left[A \cup C_{\sigma}\right.$ is r.e.], and

(ii) $\left(\forall W \supseteq A\right.$ r.e.) $\left(\exists S \subseteq T\right.$ finite) $\left[W={ }^{*} A \cup \bigcup_{\sigma \in S} C_{\sigma}\right]$,

where $C_{\sigma}=\left\{a_{\tau} \mid \tau \in T \wedge \tau \supseteq \sigma\right\}$ is the "cone" of elements of $\bar{A}$ "above" $a_{\sigma}$.

The idea is now to reduce index set proofs to proofs about trees by the above correspondence between trees $T$ and r.e. sets $A_{T}$. 
Using Lachlan's construction as a starting point, we can break up an index set classification into easier parts. Suppose we are trying to show that $\left(\Sigma_{n}, \Pi_{n}\right) \leq_{1}$ (A, B) for certain disjoint index sets $\mathrm{A}$ and $\mathrm{B}$ which are closed modulo finite sets, i.e., which satisfy

$$
e \in \mathrm{A} \wedge W_{e}={ }^{*} W_{i} \rightarrow i \in \mathrm{A}
$$

and likewise for B. (The technique works just as well if we replace the integer $n$ by a recursive ordinal $\alpha$.) Then it suffices to establish the following two lemmas:

(I) Correspondence Lemma: The mapping index of $T \mapsto$ index of $A_{T}$ maps the $\Sigma_{2}$-trees of $S$ into $A$, and the $\Sigma_{2}$-trees of $\tau$ into $B$, for certain disjoint classes of index sets of binary $\Delta_{3}$-trees $S, \tau$.

(II) Reduction Lemma: A recursive function $f$ maps $C$ into the set of recursive trees of $S$ and $\bar{C}$ into the set of recursive trees of $\tau$.

Here $C$ is a $\Sigma_{m}$-complete set (where $2+m=n$ ), and we require that membership of $T$ in $S$ and $\tau$ only depends on $[T]$, namely, for $\Delta_{3}$-trees $T$ and $\tilde{T}$,

$$
T \in S \wedge[\tilde{T}]=[T] \rightarrow \tilde{T} \in S,
$$

and likewise for $\tau$.

Once we have established (I) and (II), we can complete the proof of the index set classification as follows:

LEMMA. (i) We can relativize the construction of $f$ to $\varnothing^{\prime \prime}$ to obtain a recursive function $\tilde{f}$ mapping a $\Sigma_{m}^{\varnothing^{\prime \prime}}$-complete (i.e., $\Sigma_{n}$-complete) set $\tilde{C}$ to the $\Delta_{1}^{\varnothing^{\prime \prime}}$ trees (i.e., $\Delta_{3}$-trees) of $S$, and the complement of $\tilde{C}$ to the $\Delta_{3}$-trees of $\tau$.

(ii) We can approximate the $\Delta_{3}$-trees $\tilde{T}$ obtained in (i) by $\Sigma_{2}$-trees $\hat{T}$ with $[\tilde{T}]=$ $[\hat{T}]$, and denote this approximation of $\tilde{f}$ by $\hat{f}$.

PROOF. (i) Straightforward relativization of the construction of $f$ first yields a function $g \leq_{T} \varnothing^{\prime \prime}$. Now it is easy to find the desired partial recursive function $\tilde{f}$ such that $W_{\tilde{f}(e)}^{\varnothing^{\prime \prime}}=W_{g(e)}^{\varnothing^{\prime \prime}}$ (where these sets code the trees) by "pushing the oracle of the index function into the main oracle". Since $g$ is total, so is $\tilde{f}$.

(ii) Notice that for a $\Delta_{3}$-tree (i.e., $\Delta_{2}^{\varnothing^{\prime}}$-tree) $\tilde{T}$, there is a function $h \leq_{T} \varnothing^{\prime}$ such that $\sigma \in \tilde{T}$ iff $\lim _{s} h(\sigma, s)=1$, and $\sigma \notin \tilde{T}$ iff $\lim _{s} h(\sigma, s)=0$. Now enumerate $\hat{T}$ (relative to $\varnothing^{\prime}$ ) by putting $\sigma$ into $\hat{T}$ at stage $s$ if

$$
|\sigma| \leq s \wedge(\forall n \leq|\sigma|)[h(\sigma \uparrow n, s)=1] .
$$

Now the composition of $\hat{f}$ with the mapping index of $T \mapsto$ index of $A_{T}$ yields the desired reduction $\left(\Sigma_{n}, \Pi_{n}\right) \leq_{1}(\mathrm{~A}, \mathrm{~B})$.

Three typical examples of a correspondence as in (I) are the following: A finite tree $T$ (i.e., $[T]=\varnothing$ ) corresponds to a cofinite set $A_{T}$. A $\Sigma_{2}$-tree with exactly one infinite path corresponds to a maximal set $A_{T}$. A perfect tree $T$ is a tree such that for all $\sigma \in T$, there are $\tau_{1}, \tau_{2} \in T$ such that $\sigma \subset \tau_{1}, \tau_{2}$ and $\tau_{1} \mid \tau_{2}$. A perfect $\Sigma_{2}$-tree corresponds to an atomless hyperhypersimple set $A_{T}$. (We will give a proof below for the latter two correspondences.)

In the Reduction Lemmas below, since the construction is recursive we will ensure that the tree $T$ constructed is recursive by letting $T_{s}=T \cap 2^{\leq s}$, where $T_{s}$ is the part of $T$ constructed by the end of stage $s$. 


\section{A warm-up theorem.}

DEFINITION. Let $A$ be a coinfinite r.e. set.

(i) $A$ is maximal if for all r.e. sets $W \supseteq A$, either $W={ }^{*} A$ or $W={ }^{*} \omega$.

(ii) $A$ is quasimaximal if it is a finite intersection of maximal sets.

(iii) $A$ is atomless if it has no maximal superset.

(iv) $A$ is atomic if it has no atomless superset.

(v) $A$ is hyperhypersimple if $\mathcal{L}(A)$, the lattice of r.e. supersets of $A$, forms a Boolean algebra. (By Lachlan [La68], this is equivalent to the original definition.)

Notice that a coinfinite r.e. set having no atomic superset is the same as an atomless set, so the hierarchy collapses at that level.

Proposition. The index sets of maximal, quasimaximal, atomless, and atomic sets are $\Pi_{4}, \Sigma_{5}, \Pi_{5}$, and $\Pi_{6}$, respectively.

ProOF. By the fact that Max is $\Pi_{4}$ and the usual Tarski-Kuratowski algorithm.

We denote these index sets by Max, QMax, Atomless, and Atomic, respectively. Our machinery now allows an easy classification of these four index sets:

THEOREM A. The following reductions hold:

(i) $\left(\Pi_{4}, \Sigma_{4}\right) \leq_{1}(\operatorname{Max}$, QMax - Max);

(ii) $\left(\Sigma_{5}, \Pi_{5}\right) \leq_{1}$ (QMax, Atomless); and

(iii) $\Pi_{6} \leq_{1}$ Atomic.

COROllary. (a) (Lachlan, D. A. Martin, R. W. Robinson, Yates (unpublished); later appearing in Tulloss [Tu71]) The index set of maximal sets is $\Pi_{4}$-complete.

(b) The index set of quasimaximal sets is $\Sigma_{5}$-complete.

(c) (Jockusch) The index set of atomless sets is $\Pi_{5}$-complete.

(d) The index set of atomic sets is $\Pi_{6}$-complete.

PROOF OF THEOREM A. We have to establish (I) and (II) above for our machinery to apply. Call $T$ essentially perfect if $\operatorname{Ext}(T)$ is a perfect tree, i.e., if there is a 1-1 map $e$ from $2^{<\omega}$ into the extendible part $\operatorname{Ext}(T)$ of $T$ such that

(a) $\left(\forall \sigma, \tau \in 2^{<\omega}\right)[\sigma \subset \tau \leftrightarrow e(\sigma) \subset e(\tau)]$, and

(b) $(\forall \rho \in \operatorname{Ext}(T))\left(\exists \sigma \in 2^{<\omega}\right)[\rho \subseteq e(\sigma)]$.

We define four classes of trees:

$$
\begin{aligned}
& \tau_{1}=\left\{T \subseteq 2^{<\omega} \text { tree }||[T] \mid=1\right\}, \\
& \tau_{2}=\left\{T \subseteq 2^{<\omega} \text { tree } \mid[T] \neq \varnothing, \text { finite }\right\}, \\
& \tau_{3}=\left\{T \subseteq 2^{<\omega} \text { tree } \mid T \text { is essentially perfect }\right\}, \\
& \tau_{4}=\left\{T \subseteq 2^{<\omega} \text { tree } \mid[T] \neq \varnothing \wedge(\forall \sigma \in T)[T(\sigma) \text { is not essentially perfect }]\right\} .
\end{aligned}
$$

CORRESPONDENCE Lemma. Let $T \subseteq 2^{<\omega}$ be a $\Sigma_{2}$-tree. Then:

(i) If $T \in \tau_{1}$ then $A_{T}$ is maximal, and conversely.

(ii) If $T \in \tau_{2}$ then $A_{T}$ is quasimaximal.

(iii) If $T \in \tau_{3}$ then $A_{T}$ is atomless.

(iv) If $T \in \tau_{4}$ then $A_{T}$ is atomic, and conversely. 
Proof. (i) Let $W \supseteq A_{T}$ be an r.e. superset. Then $W={ }^{*} A_{T} \cup \bigcup_{\sigma \in S} C_{\sigma}$ for some finite set $S \subseteq T$. If $S \cap \operatorname{Ext}(T)=\varnothing$ then $W={ }^{*} A_{T}$, and, since $\left.\| T\right] \|=1$, if $S \cap \operatorname{Ext}(T) \neq \varnothing$ then $W={ }^{*} \omega$. So $A_{T}$ is maximal. The converse is shown analogously.

(ii) Similar to (i).

(iii) Suppose $W \supseteq A_{T}$ is a maximal superset. Then $W={ }^{*} A_{T} \cup \bigcup_{\sigma \in S} C_{\sigma}$ for some finite set $S \subseteq T$. Since $W$ is coinfinite there is some $\sigma_{0} \in \operatorname{Ext}(T)$ such that $C_{\sigma_{0}} \cap W=\varnothing$. Let $\tau_{0} \in 2^{<\omega}$ be such that $\sigma_{0} \subseteq e\left(\tau_{0}\right)$. Then $W \subset_{\infty} W \cup$ $C_{e\left(\tau_{0}\right.} \widehat{\langle 0\rangle)} C_{\infty} W \cup C_{e\left(\tau_{0}\right)}$, contradicting $W$ 's maximality.

(iv) Suppose $W \supseteq A_{T}$ is an atomless superset. Then $W={ }^{*} A_{T} \cup \bigcup_{\sigma \in S} C_{\sigma}$ for some finite set $S \subseteq T$. Since $W$ is coinfinite there is some $\sigma_{0} \in \operatorname{Ext}(T)$ such that $C_{\sigma_{0}} \cap W=\varnothing$. Let

$$
W_{0}=A_{T} \cup \bigcup_{\substack{|\sigma|=\left|\sigma_{0}\right| \\ \sigma \in T-\left\{\sigma_{0}\right\}}} C_{\sigma} .
$$

Then $W_{0}$ is coinfinite and $W_{0} \supseteq^{*} W$, so $W_{0}$ is also atomless. We will show that $T\left(\sigma_{0}\right)$ is essentially perfect to reach a contradiction. Let $T_{0}=\operatorname{Ext}\left(T\left(\sigma_{0}\right)\right)$. It suffices to show that, for all $\tau \in T_{0}$, there exist $\tau_{1}, \tau_{2} \in T_{0}$ such that $\tau \subset \tau_{1}, \tau_{2}$ and $\tau_{1} \mid \tau_{2}$. Suppose $\tau_{0} \in T_{0}$ does not admit such a splitting. Then

$$
W_{1}=A_{T} \cup \bigcup_{\substack{|\tau|=\left|\tau_{0}\right| \\ \tau \in T_{0}-\left\{\tau_{0}\right\}}} C_{\sigma_{0}} \widehat{\tau}
$$

is maximal by an argument similar to (i).

Conversely, assume that $T\left(\sigma_{0}\right)$ is essentially perfect for some $\sigma_{0}$. Then

$$
W=A_{T} \cup \bigcup_{\substack{|\sigma|=\left|\sigma_{0}\right| \\ \sigma \in T-\left\{\sigma_{0}\right\}}} C_{\sigma}
$$

is an atomless superset of $A_{T}$ by (iii).

REDUCTION LEMMA. We have the following reductions (where all images of the reducing maps are recursive trees):

(i) $\left(\Pi_{2}, \Sigma_{2}\right) \leq_{1}\left(\tau_{1}, \tau_{2}-\tau_{1}\right)$,

(ii) $\left(\Sigma_{3}, \Pi_{3}\right) \leq_{1}\left(\tau_{2}, \tau_{3}\right)$, and

(iii) $\Pi_{4} \leq_{1} \tau_{4}$.

PrOOF. (i) We choose Inf and Fin, the index sets of infinite and finite r.e. sets, respectively, as $\Pi_{2}$ - and $\Sigma_{2}$-complete index sets. We will build a reduction $k \mapsto T_{k}$ such that $k \in \operatorname{Inf}$ implies $T_{k} \in \tau_{1}$, and $k \in$ Fin implies $T_{k} \in \tau_{2}-\tau_{1}$. Fix $k$. At stage 0 , let $T_{k, 0}=\{\varnothing\}$; at stage 1 , we put $\langle 0\rangle$ and $\langle 1\rangle$ into $T_{k, 1}$. At a stage $s \geq 2$, if $W_{k, s} \neq W_{k, s-1}$, we put $\left\langle 0^{s}\right\rangle$ and $\left\langle 0^{s-1} 1\right\rangle$ into $T_{k, s}$; otherwise, we put $\tau \sim\langle 0\rangle$ into $T_{k, s}$ for the two $\tau \in T_{k, s-1}$ with $|\tau|=s-1$. Then

$$
\begin{aligned}
k \in \operatorname{Inf} & \rightarrow\left(\exists^{\infty} s\right)\left[W_{k, s} \neq W_{k, s-1}\right] \rightarrow\left[T_{k}\right]=\left\{\left\langle 0^{\omega}\right\rangle\right\} \rightarrow T_{k} \in T_{1}, \\
k \in \text { Fin } & \rightarrow\left(\exists^{<\infty} s\right)\left[W_{k, s} \neq W_{k, s-1}\right] \rightarrow\left[T_{k}\right]=\left\{\left\langle 0^{\omega}\right\rangle,\left\langle 0^{s_{0}-1} 10^{\omega}\right\rangle\right\} \\
& \rightarrow T_{k} \in \tau_{2}-\tau_{1},
\end{aligned}
$$

where $s_{0}=\max \left\{s \mid W_{k, s} \neq W_{k, s-1}\right\}$. 
(ii) We choose Cof and Coinf, the index sets of cofinite and coinfinite r.e. sets, respectively, as $\Sigma_{3}$ - and $\Pi_{3}$-complete index sets. We will again build a reduction $k \mapsto T_{k}$ such that $k \in$ Cof implies $T_{k} \in \tau_{2}$, and $k \in$ Coinf implies $T_{k} \in \tau_{3}$. Fix $k$ and let $\overline{W_{k, s}}=\left\{w_{k, s}^{0}<w_{k, s}^{1}<w_{k, s}^{2}<\cdots\right\}$. Let $\left\{\mu_{\sigma}\right\}_{\sigma \in 2<\omega}$ be a sequence of markers. At stage 0 , let $n_{0}=0$, let $\mu_{\varnothing, 0}=\varnothing$, let all other markers be undefined, and put $\varnothing$ into $T_{k, 0}$. At a stage $s>0$, let $n_{s}=\min \left(\left\{n_{s-1}+1\right\} \cup\left\{n \mid w_{k, s-1}^{n} \neq\right.\right.$ $\left.\left.w_{k, s}^{n}\right\}\right)$. For $|\sigma|<n_{s}$, let $\mu_{\sigma, s}=\mu_{\sigma, s-1}$. For $|\sigma|=n_{s}$, let $\mu_{\sigma, s}$ be equal to some string $\tau$ with $|\tau|=s, \tau^{-} \in T_{k, s-1}$, and $\tau \supset \mu_{\sigma^{-, s}}$ where $\rho^{-}=\rho \uparrow(|\rho|-1)$, and put all these $\tau$ into $T_{k, s}$. For $|\sigma|>n_{s}$, let $\mu_{\sigma, s}$ be undefined.

Now assume that $W_{k}$ is cofinite. Then there is some (least) $\tilde{n}$ such that $\lim _{s} w_{k, s}^{\tilde{n}}$ $=\infty$, so $\lim _{s}\left|\mu_{\sigma, s}\right|=\infty$ for all $\sigma$ with $|\sigma| \geq \tilde{n}$. But then $\liminf \operatorname{in}_{s}\left|T_{k} \cap 2^{s}\right|=2^{\tilde{n}}$, so $\left[T_{k}\right]$ is finite. $\left[T_{k}\right]$ is nonempty by König's Lemma since for all $s, T_{k} \cap 2^{s} \neq \varnothing$. Thus $T_{k} \in \tau_{2}$.

On the other hand, if $W_{k}$ is coinfinite, then $\lim _{s} w_{k, s}^{n}<\infty$ exists for all $n$, so $\lim _{s} n_{s}=\infty$. We can thus define, for all $n$, a stage $s_{n}=\max \left\{s \mid n_{s}=n\right\}$. Therefore, $\lim _{s} \mu_{\sigma, s}=\mu_{\sigma}$ exists for all $\sigma \in 2^{<\omega}$. The mapping $\sigma \mapsto \mu_{\sigma}$ now shows that $T_{k}$ is essentially perfect.

(iii) The final part of the proof is a first example of how the uniformity of the construction can be used to yield more and more complicated index set results.

There is a recursive function $g$ such that

$$
\begin{aligned}
& k \in \varnothing^{(4)} \leftrightarrow(\exists i)\left[W_{g(k, i)} \text { coinfinite }\right], \text { and } \\
& k \notin \varnothing^{(4)} \leftrightarrow(\forall i)\left[W_{g(k, i)} \text { cofinite }\right] .
\end{aligned}
$$

Fix $k$. At stage 0 , we let $T_{k, 0}=\{\varnothing\}$. At a stage $s>0$, put $\left\langle 0^{s}\right\rangle$ and $\left\langle 0^{s-1} 1\right\rangle$ into $T_{k, s}$ and start the construction described in part (ii) but above $\left\langle 0^{s-1} 1\right\rangle$ in place of $\varnothing$ and using $W_{g(k, s-1)}$ in place of $W_{k}$.

Now, if $k \notin \varnothing^{(4)}$, then for all $i, W_{g(k, i)}$ is cofinite, so $\left[T_{k}\left(\left\langle 0^{i} 1\right\rangle\right)\right]$ is finite for all $i$ by (ii), and therefore $T_{k}(\sigma)$ is not essentially perfect for any $\sigma \in T_{k}$. Thus $T_{k} \in \tau_{4}$.

On the other hand, if $k \in \varnothing^{(4)}$, then $W_{g(k, i)}$ is coinfinite for some $i$, so, again by (ii), $\left[T_{k}\left(\left\langle 0^{i} 1\right\rangle\right)\right]$ is essentially perfect. Thus $T_{k} \notin \tau_{4}$.

This establishes Theorem A by our machinery.

3. The main theorem. Call a set $A \subseteq \omega 0$-atomic iff $|\bar{A}| \leq 1$. Then a set $B$ is cofinite iff $B$ is in the filter generated by the 0 -atomic sets. A set $C$ is maximal iff its equivalence class is a coatom of the lattice of r.e. sets modulo the cofinite filter. A coinfinite set $D$ is quasimaximal iff $D$ is in the filter in $\mathcal{E}$ generated by the maximal sets, etc. This alternation of generating a filter and considering the coatoms leads to the following definition:

DEFInition. Let $A$ be a hyperhypersimple or cofinite set, $\alpha$ an ordinal, and $\lambda$ a limit ordinal. Then:

(i) $A$ is 0 -atomic if $|\bar{A}| \leq 1$;

(ii) $A$ is $\alpha$-quasiatomic if $A$ is a finite intersection of $\alpha$-atomic sets, i.e., if $A$ is in the filter generated by the $\alpha$-atomic sets;

(iii) $A$ is $(\alpha+1)$-atomic if for all r.e. sets $W \supseteq A, W$ or $A \cup \bar{W}$ is $\alpha$-quasiatomic, i.e., if $A$ is $\alpha$-quasiatomic or its equivalence class is a coatom of the lattice of r.e. sets modulo the $\alpha$-quasiatomic filter (notice here and in (v) that $A \cup \bar{W}$ is r.e. if $A$ is hyperhypersimple); 
(iv) $A$ is $<\lambda$-atomic if $A$ is $\alpha$-atomic for some $\alpha<\lambda$, i.e., if $A$ is in the filter generated by the $\alpha$-atomic sets for $\alpha<\lambda$;

(v) $A$ is $\lambda$-atomic if for all r.e. sets $W \supseteq A, W$ or $A \cup \bar{W}$ is $<\lambda$-quasiatomic, i.e., if $A$ is $<\lambda$-quasiatomic or its equivalence class is a coatom of the lattice of r.e. sets modulo the $<\lambda$-quasiatomic filter.

The notions of $\alpha$-atomic, $\alpha$-quasiatomic, and $<\lambda$-atomic are natural generalizations of the notions of cofinite sets, maximal sets, and quasimaximal sets. Namely, $A$ is cofinite iff $A$ is 0-quasiatomic; $A$ is maximal (or cofinite) iff $A$ is 1-atomic; and $A$ is quasimaximal (or cofinite) iff $A$ is 1-quasiatomic.

Let $\mathrm{At}_{\alpha}, \mathrm{QAt}_{\alpha}$, and $\mathrm{At}_{<\lambda}$ denote the index sets of $\alpha$-atomic, $\alpha$-quasiatomic, and $<\lambda$-atomic sets, respectively.

The importance of the above definition lies in the correspondence of these properties with the Cantor-Bendixson rank of binary trees, as explained below. This correspondence allows the classification of their index sets, yielding a family of index sets of properties $\mathbf{L}_{\omega_{1}, \omega}$-definable over $\mathcal{E}$, which goes all the way through the hyperarithmetical hierarchy.

In the following, we will use ordinal arithmetic to compute expressions like $2 \alpha+2$, etc. A set of integers is $\Sigma_{\lambda+n}\left(\Pi_{\lambda+n}\right)$ (for $\lambda$ a recursive limit ordinal, $n \in \omega-\{0\}$ ) iff it is $\Sigma_{n}^{\varnothing^{(\lambda)}}\left(\Pi_{n}^{\varnothing^{(\lambda)}}\right)$. We use Rogers's book [Ro67] for the background on recursive ordinals. He defines a system of ordinal notations $|\cdot|: 0 \rightarrow \omega_{1}^{\mathrm{CK}}$ from Kleene's $0 \subseteq \omega$ into the set of recursive ordinals as well as a partial order $<_{0}$ on 0 by

$$
\begin{aligned}
& |1|=0, \\
& |x|=\alpha \rightarrow\left|2^{x}\right|=\alpha+1, \text { and } z \leq_{0} x \rightarrow z<_{0} 2^{x}, \\
& \left\{\varphi_{y}(n)\right\}_{n \in \omega} \text { a }<_{0} \text {-increasing sequence and } \sup _{n}\left|\varphi_{y}(n)\right|=\alpha \\
& \quad \rightarrow\left|3 \cdot 5^{y}\right|=\alpha \text {, and }(\exists n)\left[z<_{0} \varphi_{y}(n)\right] \rightarrow z<_{0} 3 \cdot 5^{y} .
\end{aligned}
$$

The hyperarithmetical hierarchy $H: 0 \rightarrow 2^{\omega}$ is then defined by

$$
\begin{aligned}
H(1) & =\varnothing, \\
H\left(2^{x}\right) & =(H(x))^{\prime}, \\
H\left(3 \cdot 5^{y}\right) & =\left\{\langle u, v\rangle \mid u \in H(v) \wedge v<_{0} 3 \cdot 5^{y}\right\} .
\end{aligned}
$$

Now $|x| \leq|y|$ implies $H(x) \leq_{T} H(y)$. In particular, the Turing degree of $H\left(3 \cdot 5^{y}\right)$ does not depend upon the specific notation for a limit ordinal $\lambda=\left|3 \cdot 5^{y}\right|$. Thus the definition of $\Sigma_{\lambda+n}$ and $\Pi_{\lambda+n}$ does not depend upon which $H\left(3 \cdot 5^{y}\right)$ with $\left|3 \cdot 5^{y}\right|=\lambda$ we use for $\varnothing^{(\lambda)}$. (Recall also that for any $y \in O,\left\{x \mid x<_{0} y\right\}$ is r.e. uniformly in y.)

The following theorem generalizes Theorem A (i) and (ii) to the hyperarithmetical hierarchy. We can do so by bounding the Cantor-Bendixson rank of the associated trees more carefully.

THEOREM B. Let $\alpha$ be a recursive ordinal and $\lambda$ a recursive limit ordinal. Then:

(i) $\left(\Pi_{2 \alpha+2}, \Sigma_{2 \alpha+2}\right) \leq_{1}\left(\mathrm{At}_{\alpha}, \mathrm{QAt}_{\alpha}-\mathrm{At}_{\alpha}\right)$;

(ii) $\left(\Sigma_{2 \alpha+3}, \Pi_{2 \alpha+3}\right) \leq_{1}\left(\mathrm{QAt}_{\alpha}, \mathrm{At}_{\alpha+1}-\mathrm{QAt}_{\alpha}\right)$; and

(iii) $\left(\Sigma_{\lambda+1}, \Pi_{\lambda+1}\right) \leq_{1}\left(\mathrm{At}_{<\lambda}, \mathrm{At}_{\lambda}-\mathrm{At}_{<\lambda}\right)$. 
COROLlaRY 1. (a) $\mathrm{At}_{\alpha}$ is $\Pi_{2 \alpha+2}$-complete;

(b) $\mathrm{QAt}_{\alpha}$ is $\Sigma_{2 \alpha+3}$-complete; and

(c) $\mathrm{At}_{<\lambda}$ is $\Sigma_{\lambda+1}$-complete.

PROOF. By Theorem B and the fact that $\mathrm{At}_{\alpha}, \mathrm{QAt}_{\alpha}$, and $\mathrm{At}_{<\lambda}$ are $\Pi_{2 \alpha+2}$, $\Sigma_{2 \alpha+3}$, and $\Sigma_{\lambda+1}$, respectively, by the Tarski-Kuratowski algorithm. E.g.,

$$
\begin{aligned}
x \in \mathrm{At}_{0} & \leftrightarrow(\forall y)\left[y \in W_{x} \vee(\forall z>y)\left[z \in W_{x}\right]\right] \\
& \leftrightarrow(\forall y)\left[\Sigma_{1} \vee \Pi_{2}\right],
\end{aligned}
$$

and

$$
x \in \mathrm{At}_{<\omega} \leftrightarrow(\exists n)\left[x \in \mathrm{At}_{n}\right] \leftrightarrow(\exists n) R(x, n),
$$

where $R$ is a $\varnothing^{(\omega)}$-recursive predicate.

Corollary 2. (a) (Lachlan, D. A. Martin, R. W. Robinson, Yates (unpublished); later appearing in Tulloss [Tu71]) The index set of maximal sets is $\Pi_{4}$-complete.

(b) The index set of quasimaximal sets is $\Sigma_{5}$-complete.

ProOF. Set $\alpha=1$ in Corollary 1.

PROOF OF THEOREM B. The proof for the 0-atomic case does not fit into our machinery but follows easily from $\left(\Sigma_{2}, \Pi_{2}\right) \leq_{1}$ (Fin, Tot). Using the machinery, we again have to prove a Correspondence Lemma and a Reduction Lemma.

Recall the definitions of Cantor-Bendixson derivative and Cantor-Bendixson rank. The Cantor-Bendixson derivative of a tree $T \subseteq 2^{<\omega}$ is $T$ minus its isolated paths, i.e.,

$$
D(T)=\left\{\sigma \in \operatorname{Ext}(T) \mid\left(\exists \tau_{1}, \tau_{2} \in \operatorname{Ext}(T)\right)\left[\sigma \subset \tau_{1}, \tau_{2} \wedge \tau_{1} \mid \tau_{2}\right]\right\} .
$$

We also define its iterates:

$$
\begin{aligned}
D^{0}(T) & =T, \\
D^{\alpha+1}(T) & =D\left(D^{\alpha}(T)\right), \\
D^{\lambda}(T) & =\bigcap_{\alpha<\lambda} D^{\alpha}(T),
\end{aligned}
$$

where $\alpha$ is an ordinal, $\lambda$ is a limit ordinal. Then the Cantor-Bendixson rank of $T$ is

$$
\rho(T)= \begin{cases}-1 & \text { if } T \text { is finite } \\ \min \left\{\alpha \mid D^{\alpha+1}(T) \text { finite }\right\} & \text { if } T \text { is infinite } \\ \infty=\min \left\{\alpha||\left[D^{\alpha}(T)\right] \mid \text { finite }\right\} & \text { and this ordinal exists } \\ \text { otherwise }\end{cases}
$$

It is a well-known fact that $D^{\alpha}(T)=D^{\beta}(T)$ for any uncountable ordinals $\alpha$ and $\beta$; and that $D^{\lambda}(T)$ finite for some limit ordinal $\lambda$ implies $D^{\alpha}(T)$ finite for some $\alpha<\lambda$ by compactness.

These definitions lead to the

CORRESPONDENCE LEMMA. Let $\alpha$ be a recursive ordinal, $T \subseteq 2^{<\omega}$ a $\Sigma_{2}$-tree. Then:

(i) $\rho(T)=-1$ iff $A_{T}$ is 0-quasiatomic;

(ii) $\left|\left[D^{\alpha}(T)\right]\right| \leq 1$ iff $A_{T}$ is $(1+\alpha)$-atomic; and

(iii) $\rho(T) \leq \alpha$ iff $A_{T}$ is $(1+\alpha)$-quasiatomic. 
PrOOF. By induction on $\alpha$ :

(i). $\rho(T)=-1$ iff $T$ is finite iff $A_{T}$ is cofinite iff $A_{T}$ is 0-quasiatomic.

(ii) $\alpha=0$. By (i) and the Correspondence Lemma for Theorem A.

(ii) $)_{\alpha} \rightarrow$ (iii) $)_{\alpha}$. Assume (ii) for an ordinal $\alpha$.

Suppose first that $\rho(T) \leq \alpha$. Then $\left[D^{\alpha}(T)\right]$ is finite, say,

$$
\left[D^{\alpha}(T)\right] \subseteq\left\{p_{1}, p_{2}, \ldots, p_{n}\right\} .
$$

Let $k$ be large enough such that $i \neq j$ implies $p_{i} \uparrow k \neq p_{j} \uparrow k$. Then $\left|\left[D^{\alpha}\left(\sigma^{\wedge} T(\sigma)\right)\right]\right|$ $\leq 1$ for all $\sigma \in T \cap 2^{k}$. By induction,

$$
A_{\sigma}=\operatorname{def} A_{T} \cup \bigcup_{\substack{|\tau|=|\sigma|, \tau \neq \sigma \\ \tau \in T}} C_{\tau}
$$

is $(1+\alpha)$-atomic, thus $A_{T}={ }^{*} \bigcap_{\sigma \in T \cap 2^{k}} A_{\sigma}$ is $(1+\alpha)$-quasiatomic.

On the other hand, if $A_{T}$ is $(1+\alpha)$-quasiatomic then $A_{T}=\bigcap_{i=1}^{n} A_{i}$ for a finite set of $(1+\alpha)$-atomic sets $A_{1}, A_{2}, \ldots, A_{n}$. For each $i$, let $A_{i}={ }^{*} A_{T} \cup \bigcup_{\sigma \in S_{i}} C_{\sigma}$ for some finite set $S_{i} \subseteq T$, and let $T_{i}=T-\bigcup_{\sigma \in S_{i}} \sigma^{\frown} T(\sigma)$. Then $\bigcup_{i=1}^{n} T_{i}={ }^{*} T$, and, by induction, $\left[D^{\alpha}\left(T_{i}\right)\right] \subseteq\left\{p_{i}\right\}$ for some $p_{i} \in 2^{\omega}$. Thus $\left[D^{\alpha}(T)\right] \subseteq\left\{p_{1}, p_{2}, \ldots, p_{n}\right\}$ is finite, and $\rho(T) \leq \alpha$.

(iii) $<\alpha \rightarrow(\text { ii })_{\alpha}$. Assume $\alpha>0$, and that (iii) holds for all ordinals less than $\alpha$. Without loss of generality, let $\alpha$ be a successor ordinal and put $\beta+1=\alpha$ (if $\alpha$ is a limit ordinal, replace $\beta$ by $<\alpha$ throughout this part of the proof).

Suppose first that $\left|\left[D^{\alpha}(T)\right]\right| \leq 1$, say, $\left[D^{\alpha}(T)\right] \subseteq\{p\}$. If $W \supseteq A_{T}$ is r.e. then $W={ }^{*} A_{T} \cup \bigcup_{\sigma \in S} C_{\sigma}$ for some finite set $S \subseteq T$ (assume that all $\sigma \in S$ are of the same length, say, $k)$. Let $S_{0}=\left(2^{k}-S\right) \cap T$, and put $W_{0}=A_{T} \cup \bigcup_{\sigma \in S_{0}} C_{\sigma}$. Then $W_{0}$ is the relative complement (w.r.t. $A_{T}$ ) of $W$ (modulo a finite set). Without loss of generality, suppose that $p \uparrow k \in S_{0}$ (the other case is symmetric). Then $T_{0}=T-\bigcup_{\sigma \in S_{0}} C_{\sigma}$, the tree associated with $W_{0}$, satisfies $\left[D^{\alpha}\left(T_{0}\right)\right]={ }^{*} \varnothing$, and so $W_{0}$ is $(1+\beta)$-quasiatomic. Thus $A_{T}$ is $(1+\alpha)$-atomic.

On the other hand, let $A_{T}$ be $(1+\alpha)$-atomic. Suppose for the sake of contradiction that $\left[D^{\alpha}(T)\right]$ contains two distinct infinite paths, say, $p_{1}$ and $p_{2}$. Let $k$ be large enough that $p_{1} \uparrow k \neq p_{2} \uparrow k$; let $S_{1}$ and $S_{2}$ be such that $S_{1} \sqcup S_{2}=2^{k} \cap T, p_{1} \uparrow k \in S_{1}$, and $p_{2} \uparrow k \in S_{2}$; and let $W_{1}=A_{T} \cup \bigcup_{\sigma \in S_{1}} C_{\sigma}$ and $W_{2}=A_{T} \cup \bigcup_{\sigma \in S_{2}} C_{\sigma}$. Thus $W_{1}$ and $W_{2}$ are relative complements (w.r.t. $A$ ) to each other (modulo a finite set). Then for both $T_{1}=T-\bigcup_{\sigma \in S_{1}} C_{\sigma}$ and $T_{2}=T-\bigcup_{\sigma \in S_{2}} C_{\sigma},\left[D^{\alpha}\left(T_{1}\right)\right]$ and $\left[D^{\alpha}\left(T_{2}\right)\right]$ are nonempty (namely, $p_{1} \in\left[D^{\alpha}\left(T_{2}\right)\right]$ and $p_{2} \in\left[D^{\alpha}\left(T_{1}\right)\right]$ ), and thus, by induction, neither of their associated r.e. sets $W_{1}$ and $W_{2}$ is $(1+\beta)$-quasiatomic, a contradiction.

4. The Reduction Lemma for the main theorem. Let $\alpha$ be a recursive ordinal. We define

$$
\begin{aligned}
S_{\alpha} & =\left\{T \in 2^{<\omega} \text { tree }||\left[D^{\alpha}(T)\right] \mid \leq 1\right\}, \\
\tau_{\alpha} & =\left\{T \in 2^{<\omega} \text { tree } \mid \rho(T) \leq \alpha\right\}(\text { allow } \alpha=-1), \\
\tau_{<\alpha} & =\bigcup_{\beta<\alpha} \tau_{\beta} .
\end{aligned}
$$


It remains to prove the

REDUCTION LEMMA. Let $\alpha$ be a recursive ordinal and $\lambda$ a recursive limit ordinal. Then:

(i) $\left(\Pi_{2 \alpha+2}, \Sigma_{2 \alpha+2}\right) \leq_{1}\left(S_{\alpha}, \tau_{\alpha}-S_{\alpha}\right)$;

(ii) $\left(\Sigma_{2 \alpha+3}, \Pi_{2 \alpha+3)} \leq_{1}\left(\tau_{\alpha}, S_{\alpha+1}-\tau_{\alpha}\right)\right.$ (also allow $\left.\alpha=-1\right)$; and

(iii) $\left(\Sigma_{\lambda+1}, \Pi_{\lambda+1}\right) \leq_{1}\left(\tau_{<\lambda}, S_{\lambda}-\tau_{<\lambda}\right)$.

Notice that this lemma is an extension of the Reduction Lemma for Theorem A. Let LOR be the class of limit ordinals.

PROOF. All constructions will be uniform in an ordinal notation for $\alpha$ (or $\lambda$ ), so we can use transfinite induction and the following four statements for $\alpha, \lambda \geq 0$ :

(A) $\left(\Sigma_{1}, \Pi_{1}\right) \leq_{1}\left(\tau_{-1}, S_{0}-\tau_{-1}\right)$;

(B) $\left(\Sigma_{2 \alpha+1}, \Pi_{2 \alpha+1}\right) \leq_{1}\left(\tau_{<\alpha}, S_{\alpha}-\tau_{<\alpha}\right) \rightarrow\left(\Sigma_{2 \alpha+3}, \Pi_{2 \alpha+3}\right) \leq_{1}\left(\tau_{\alpha}, S_{\alpha+1}-\tau_{\alpha}\right)$;

(C) $\left(\Sigma_{2 \alpha+1}, \Pi_{2 \alpha+1}\right) \leq_{1}\left(\tau_{<\alpha}, S_{\alpha}-\tau_{<\alpha}\right) \rightarrow\left(\Pi_{2 \alpha+2}, \Sigma_{2 \alpha+2}\right) \leq_{1}\left(S_{\alpha}, \tau_{\alpha}-S_{\alpha}\right)$; and

(D) $\left(\Sigma_{\lambda+1}, \Pi_{\lambda+1}\right) \leq_{1}\left(\tau_{<\lambda}, S_{\lambda}-\tau_{<\lambda}\right)$.

Then (ii) for $\alpha=-1$ follows from (A); (ii) for $\alpha \geq 0$ and (i) follow from (ii) for $\alpha-1$ (if $\alpha \notin \mathrm{LOR}$ ) or from (iii) (if $\alpha \in \mathrm{LOR}$ ) by (B) and (C), respectively; and (iii) follows by $(\mathrm{D})$. (Notice that the proof of $(\mathrm{D})$ will require an induction argument separate from the successor ordinal case (B)-(C), as explained later.)

We will now prove $(\mathrm{A})-(\mathrm{D})$ :

(A) Given $k$, we will construct a recursive tree $T_{k}$ such that

$$
\begin{aligned}
& k \in \varnothing^{\prime} \rightarrow T_{k} \text { finite }, \\
& k \notin \varnothing^{\prime} \rightarrow\left|\left[T_{k}\right]\right|=1 .
\end{aligned}
$$

At any stage $s$, put $\left\langle 0^{s}\right\rangle$ into $T_{k, s}$ iff $\{k\}_{s}(k) \uparrow$. This construction obviously satisfies the claim.

(B) By (A) (for $\alpha=0$ ), (B) (for $\alpha \notin \operatorname{LOR} \cup\{0\}$ ), or (D) (for $\alpha \in \mathrm{LOR}$ ), we have a uniformly recursive sequence of trees $\left\{\tilde{T}_{l}\right\}_{l \in \omega}$ satisfying

$$
\begin{aligned}
& l \in \varnothing^{(2 \alpha+1)} \rightarrow\left[D^{\alpha}\left(\tilde{T}_{l}\right)\right]=\varnothing, \\
& l \notin \varnothing^{(2 \alpha+1)} \rightarrow\left|\left[D^{\alpha}\left(\tilde{T}_{l}\right)\right]\right|=1 .
\end{aligned}
$$

Now $\varnothing^{(2 \alpha+3)} \equiv_{1}$ Cof $^{\varnothing^{(2 \alpha)}}$, so, given $k$, it suffices to uniformly build a recursive tree $T_{k}$ such that

$$
\begin{aligned}
& k \in \operatorname{Cof}^{\varnothing^{(2 \alpha)}} \rightarrow\left[D^{\alpha}\left(T_{k}\right)\right] \text { finite, } \\
& k \notin \operatorname{Cof}^{\varnothing^{(2 \alpha)}} \rightarrow\left|\left[D^{\alpha+1}\left(T_{k}\right)\right]\right|=1 .
\end{aligned}
$$

Define a recursive function $f$ such that $f(k, l) \in \varnothing^{(2 \alpha+1)}$ iff $l \in W_{k}^{\varnothing^{(2 \alpha)}}$. Fix $k$. At stage 0 , put $\varnothing$ into $T_{k, 0}$. At any stage $s>0$, put $\left\langle 0^{s}\right\rangle$ and $\left\langle 0^{s-1} 1\right\rangle$ into $T_{k, s}$ and start the construction of $\tilde{T}_{f(k, s-1)}$ on top of $\left\langle 0^{s-1} 1\right\rangle$.

If $k \in \operatorname{Cof}^{\varnothing^{(2 \alpha)}}$ then $f(k, l) \notin \varnothing^{(2 \alpha+1)}$ for only finitely many $l$, say, $l_{0}$ is greater than all such $l$. Then $\left[D^{\alpha}\left(T_{k}\left(\left\langle 0^{l} 1\right\rangle\right)\right)\right]=\varnothing$ for all $l \geq l_{0}$, so $\left[D^{\alpha}\left(T_{k}\left(\left\langle 0^{l_{0}}\right\rangle\right)\right)\right] \subseteq$ $\left\{\left\langle 0^{\omega}\right\rangle\right\}$. Also $\left[D^{\alpha}\left(T_{k}\left(\left\langle 0^{l} 1\right\rangle\right)\right)\right]$ is finite for all $l<l_{0}$, so $\left[D^{\alpha}\left(T_{k}\right)\right]$ is finite. 
On the other hand, if $k \notin$ Cof $^{\varnothing^{(2 \alpha)}}$ then $f(k, l) \notin \varnothing^{(2 \alpha+1)}$ for infinitely many $l$, so $\left|\left[D^{\alpha}\left(T_{k}\left(\left\langle 0^{l} 1\right\rangle\right)\right)\right]\right|=1$ for infinitely many $l$. Thus $\left[D^{\alpha+1}\left(T_{k}\right)\right]=\left\{\left\langle 0^{\omega}\right\rangle\right\}$.

(C) The proof is similar to the proof for (B). We use the fact that $\left(\Pi_{2 \alpha+2}, \Sigma_{2 \alpha+2}\right)$ $\leq_{1}\left(\operatorname{Tot}^{\varnothing^{(2 \alpha)}}\right.$, Cotwo $\left.\varnothing^{\varnothing^{(2 \alpha)}}\right)$, where $\operatorname{Tot}^{X}$ and Cotwo ${ }^{X}$ are the index sets of total functions recursive in $X$ and functions recursive in $X$ undefined for exactly two integers, respectively.

Given $k$ and $\left\{\tilde{T}_{l}\right\}_{l \in \omega}$ as in the proof of (B), we have to uniformly build a recursive tree $T_{k}$ such that

$$
\begin{aligned}
& k \in \operatorname{Tot}^{\varnothing^{(2 \alpha)}} \rightarrow\left|\left[D^{\alpha}\left(T_{k}\right)\right]\right| \leq 1, \\
& k \in \operatorname{Cotwo}^{\varnothing^{(2 \alpha)}} \rightarrow 1<\left|\left[D^{\alpha}\left(T_{k}\right)\right]\right|<\aleph_{0} .
\end{aligned}
$$

The construction is the same as in (B).

If $k \in \operatorname{Tot}^{\varnothing^{(2 \alpha)}}$ then $f(k, l) \in \varnothing^{(2 \alpha+1)}$ for all $l$, so $\left[D^{\alpha}\left(T_{k}\left(\left\langle 0^{l} 1\right\rangle\right)\right)\right]=\varnothing$ for all $l$. Thus $\left[D^{\alpha}\left(T_{k}\right)\right] \subseteq\left\{\left\langle 0^{\omega}\right\rangle\right\}$.

On the other hand, if $k \in \operatorname{Cotwo}^{\varnothing^{(2 \alpha)}}$ then $f(k, l) \notin \varnothing^{(2 \alpha+1)}$ for exactly two distinct $l$, say, $l_{1}$ and $l_{2}$, and so $D^{\alpha}\left(T_{k}\left(\left\langle 0^{l} 1\right\rangle\right)\right)$ has exactly one infinite path for $l=l_{1}$ or $l_{2}$, and none for all other $l$. Thus $2 \leq\left|\left[D^{\alpha}\left(T_{k}\right)\right]\right| \leq 3$ (since possibly $\left.\left\langle 0^{\omega}\right\rangle \in\left[D^{\alpha}\left(T_{k}\right)\right]\right)$.

Part (D) is much harder to prove and requires some preparation.

5. The Reduction Lemma: The limit ordinal case. The first lemma generalizes a lemma by Solovay for $\lambda=\omega$ [JLSSta] to arbitrary recursive limit ordinals:

LEMMA 1 (APPROXIMATION LEMMA). Let $\lambda$ be a recursive limit ordinal and $\left\{\alpha_{n}\right\}_{n \in \omega}$ the increasing sequence with $\sup _{n} \alpha_{n}=\lambda$ given by our ordinal notation for $\lambda$ (i.e., $\left.\lambda=\left|3 \cdot 5^{x}\right|,\left|\varphi_{x}(n)\right|=\alpha_{n}\right)$. Then there is a recursive function $d$ (uniformly in a notation for $\lambda$ ) such that

$$
(\forall y)\left[y \in \varnothing^{(\lambda+1)} \leftrightarrow(\exists n)\left[d(y, n) \in \varnothing^{\left(\alpha_{n}+1\right)}\right]\right]
$$

Here $\varnothing^{(\lambda+1)}=\left(H\left(3 \cdot 5^{x}\right)\right)^{\prime}$, and $\varnothing^{\left(\alpha_{n}+1\right)}=\left(H\left(\varphi_{x}(n)\right)\right)^{\prime}$.

PROOF. Recall that there are recursive functions $h_{a, b}$ (uniformly in $a, b$ ) and r.e. sets $P_{a}$ (uniformly in $a$ ) such that

$$
\begin{gathered}
H(a) \leq_{1} H(b) \text { via } h_{a, b}\left(\text { for } a \leq_{0} b\right), \text { and } \\
P_{a}=\left\{b \mid b<_{0} a\right\} \text { for } a \in 0
\end{gathered}
$$

(See Rogers [Ro67] for details.) 


\section{Now}

(19)

$$
\begin{aligned}
y \in \varnothing^{(\lambda+1)} & \leftrightarrow\{y\}^{H\left(3 \cdot 5^{x}\right)}(y) \downarrow \\
\leftrightarrow & (\exists u, v, s)\left[\{y\}_{s}^{\left(D_{u}, D_{v}\right)}(y) \downarrow \wedge D_{u} \subseteq H\left(3 \cdot 5^{x}\right) \wedge D_{v} \cap H\left(3 \cdot 5^{x}\right)=\varnothing\right] \\
\leftrightarrow & (\exists u, v, s)\left[\{y\}_{s}^{\left(D_{u}, D_{v}\right)}(y) \downarrow \wedge\left(\forall\left\langle z_{1}, z_{2}\right\rangle \in D_{u}\right)\left[z_{1} \in H\left(z_{2}\right) \wedge z_{2}<_{0} 3 \cdot 5^{x}\right] \wedge\right. \\
\leftrightarrow & (\exists u, v, s, n)\left[\{y\}_{s}^{\left(D_{u}, D_{v}\right)}(y) \downarrow \wedge \quad\left(\forall\left\langle z_{1}, z_{2}\right\rangle \in D_{v}\right)\left[z_{1} \notin H\left(z_{2}\right) \vee z_{2} \nless_{0} 3 \cdot 5^{x}\right]\right] \\
& \left(\forall\left\langle z_{1}, z_{2}\right\rangle \in D_{u}\right)\left[h_{z_{2}, \varphi_{x}(n)}\left(z_{1}\right) \in H\left(\varphi_{x}(n)\right) \wedge z_{2} \in P_{\varphi_{x}(n), s} \wedge z_{2} \in P_{3 \cdot 5^{x}}\right] \wedge \\
& \left.\left(\forall\left\langle z_{1}, z_{2}\right\rangle \in D_{v}\right)\left[\left(h_{z_{2}, \varphi_{x}(n)}\left(z_{1}\right) \notin H\left(\varphi_{x}(n)\right) \wedge z_{2} \in P_{\varphi_{x}(n), s}\right) \vee z_{2} \notin P_{3 \cdot 5^{x}}\right]\right] \\
\leftrightarrow & (\exists n)(\exists u, v, s)\left[\Delta_{1} \wedge(\mathrm{Q})\left[\Delta_{1}^{H\left(\varphi_{x}(n)\right)} \wedge \Delta_{1} \wedge \Sigma_{1}\right] \wedge(\mathrm{Q})\left[\left(\Delta_{1}^{H\left(\varphi_{x}(n)\right)} \wedge \Delta_{1}\right) \vee \Pi_{1}\right]\right]
\end{aligned}
$$

where (Q) denotes a bounded quantifier, and $\{y\}^{\left(D_{u}, D_{v}\right)}$ that the computation uses from the oracle set $X$ at most that $z \in X$ for $z \in D_{u}$ and that $z \notin X$ for $z \in D_{v}$.

Now the matrix of the last expression is recursive in $H\left(\varphi_{x}(n)\right) \oplus \varnothing^{\prime}$, and thus the expression following $(\exists n)$ is recursive in $\left(H\left(\varphi_{x}(n+1)\right)\right)^{\prime}=\varnothing^{\left(\alpha_{n+1}+1\right)}$. This establishes the claim of the lemma.

The first try at the construction of $T_{k}$ at a limit ordinal level $\lambda$ satisfying (D) would be to build $T_{d(k, n)}^{\alpha_{n}}$ on top of $\left\langle 0^{n} 1\right\rangle$. However, we only know $\rho\left(T_{d(k, n)}^{\alpha_{n}}\right)=\alpha_{n}$ or $<\alpha_{n}$, so $\sup _{n} \rho\left(T_{d(k, n)}^{\alpha_{n}}\right)=\lambda$ is possible independent of whether $k \in \varnothing^{(\lambda+1)}$.

Our second try is to let the level $\alpha_{n}$ at which we "discover" that $k \in \varnothing^{(\lambda+1)}$ by Lemma 1, stop the higher levels by some kind of "permission" for extending branches above $\left\langle 0^{m} 1\right\rangle$ for $m>n$. However, this is hard since $T_{d(k, n)}^{\alpha_{m}}$ looks very different from $T_{d(k, n)}^{\alpha,}$, so we have to introduce a very strong kind of permission at all branchings of the much bigger tree $T_{d(k, m)}^{\alpha_{m}}$. Keeping this in mind should make the following construction seem less mysterious. This also requires a new induction argument at the successor ordinal level.

For the sake of convenience, let $\sigma\left(k_{1}, k_{2}, \ldots, k_{n}\right)=\left\langle 0^{k_{1}} 10^{k_{2}} 1 \ldots 0^{k_{n}} 1\right\rangle \in 2^{<\omega}$. For $\alpha$ a recursive ordinal, the field of the $\alpha$-strategy $F_{\alpha}$ (i.e., the largest possible tree that $T_{k}^{\alpha}$ could be) is defined by

$$
\begin{aligned}
& F_{0}=\left\{\left\langle 0^{n}\right\rangle \mid n \in \omega\right\}, \\
& F_{\alpha+1}=\left\{\sigma(n)^{\wedge} \sigma \mid \sigma \in F_{\alpha}, n \in \omega\right\} \cup F_{0}, \\
& F_{\lambda}=\left\{\sigma(n)^{\wedge} \sigma \mid \sigma \in F_{\alpha_{n}}, n \in \omega\right\} \cup F_{0} \\
& \text { for } \lambda \in \mathrm{LOR}, \lambda=\left|3 \cdot 5^{y}\right|, \alpha_{n}=\left|\varphi_{y}(n)\right| \text {. }
\end{aligned}
$$

(Notice that the $F_{\alpha}$ 's are all recursive sets, and that they $d o$ depend upon the particular ordinal notation chosen. However, since we will always fix an ordinal notation in advance this will not matter in the following.)

The ordinal $\beta_{\sigma}^{\alpha}$ associated with a branching node $\sigma$ on $F_{\alpha}$ is defined by

$$
\begin{aligned}
\beta_{\varnothing}^{\alpha} & =\alpha, \\
\beta_{\sigma \widehat{\sigma}(k)}^{\alpha} & = \begin{cases}\beta_{\sigma}^{\alpha}-1 & \text { for } \beta_{\sigma}^{\alpha} \notin \operatorname{LOR} \cup\{0\}, \\
\gamma_{k} & \text { for } \beta_{\sigma}^{\alpha}=\gamma \in \operatorname{LOR}, \gamma=\left|3 \cdot 5^{z}\right|, \gamma_{n}=\left|\varphi_{z}(n)\right|, \\
\text { undefined } & \text { for } \beta_{\sigma}^{\alpha}=0 .\end{cases}
\end{aligned}
$$


(Thus $\beta_{\sigma}^{\alpha}$ is defined exactly for all nodes $\sigma \in F_{\alpha}$ of the form $\sigma=\sigma\left(k_{1}, k_{2}, \ldots, k_{n}\right)$. The ordinals $\beta_{\sigma}^{\alpha}$ will determine the strategy above the node $\sigma$.)

The following lemma will be essential later:

LEMMA 2 (FINITE EXCEPTIONS LEMMA). For any subtree $S \subseteq F_{\alpha}$ and any infinite path $p \in[S],\{i \mid p(i)=1\}$ is finite.

PROOF. Otherwise there are $n_{1}, n_{2}, n_{3}, \ldots \in \omega$ such that $\varnothing \subset \sigma\left(n_{1}\right) \subset \sigma\left(n_{1}, n_{2}\right)$ $\subset\left(n_{1}, n_{2}, n_{3}\right) \subset \cdots \subset p$, so that all these nodes are in $S$ and thus in $F_{\alpha}$, but then $\beta_{\varnothing}^{\alpha}, \beta_{\sigma\left(n_{1}\right)}^{\alpha}, \beta_{\sigma\left(n_{1}, n_{2}\right)}^{\alpha}, \beta_{\sigma\left(n_{1}, n_{2}, n_{3}\right)}^{\alpha}, \ldots$ is an infinite descending sequence of ordinals.

We call a tree $T \subseteq F_{\alpha} \alpha$-dense (for $\alpha$ a recursive ordinal) iff

$$
\begin{aligned}
& (\forall n \in \omega \cap(\alpha+1))\left(\text { a.e. } k_{1}\right)\left(\text { a.e. } k_{2}\right) \ldots\left(\text { a.e. } k_{n}\right) \\
& \left.\qquad \rho\left(T\left(\sigma\left(k_{1}, k_{2}, \ldots, k_{n}\right)\right)\right)=\beta_{\sigma\left(k_{1}, k_{2}, \ldots, k_{n}\right)}^{\alpha}\right] .
\end{aligned}
$$

I.e., in an $\alpha$-dense tree, all appropriate subtrees of $T$ have maximal rank possible. For example, the only 0 -dense tree is $F_{0}$ itself; a tree $T \subseteq F_{1}$ is 1-dense iff $T(\sigma(n))=$ $F_{0}$ for almost all $n$, etc.

LEMMA 3 (DENSITY LEMMA). Let $\alpha>0$ be a recursive ordinal, $T \subseteq F_{\alpha} a$ tree. Then $T$ is $\alpha$-dense iff (a.e. $m)\left[T(\sigma(m))\right.$ is $\beta_{\sigma(m)}^{\alpha}$-dense $]$.

ProOF. $(\rightarrow)$ Trivial by definition.

$(\leftarrow)$ We only need to show (20) for $n=0$. Suppose that for all $m>m_{0}$, $\rho(T(\sigma(m)))=\beta_{\sigma(m)}^{\alpha}$. Since $\beta_{\sigma(m)}^{\alpha}=\alpha-1$ (for $\alpha \notin \mathrm{LOR}$ ) or $\alpha=\sup _{m} \beta_{\sigma(m)}^{\alpha}$ (for $\alpha \in \mathrm{LOR}$ ), we obtain $\rho(T)=\alpha$.

LEMMA 4 (INTERSECTION LEMMA). Let $\alpha$ be a recursive ordinal. If $T$ and $\tilde{T}$ are $\alpha$-dense, then so is $T \cap \tilde{T}$.

ProOF. By induction on $\alpha$ : For $\alpha=0$, note that $T=\tilde{T}=\left\{\left\langle 0^{m}\right\rangle \mid m \in \omega\right\}$. For $\alpha>0$, use Lemma 3 and the fact that $\beta_{\sigma(m)}^{\alpha}<\alpha$.

Notice that this would be false, for example, if we had defined $\alpha$-dense just as having rank $\alpha$. For example, then the intersection of $T, \tilde{T} \subseteq F_{1}$, both of rank 1 , could have rank 0 .

The following lemma will be essential later for showing that the nesting of trees works properly. (It is the first example of the property of trees that the subtree above a certain node $\sigma\left(k_{1}, k_{2}, \ldots, k_{n}\right)$ looks exactly as if it were constructed by itself.)

LEMMA 5 (NESTING LEMMA). Let $\beta<\alpha$ be two recursive ordinals, and let $T \subseteq F_{\beta}$ be a $\beta$-dense tree. Then $\tilde{T}=\left\{\sigma \in F_{\alpha} \mid(\forall \tau \subseteq \sigma)\left[\tau \in F_{\beta} \rightarrow \tau \in T\right]\right\}$ is $\alpha$-dense.

Proof. By induction on $\beta$ : If $\beta=0$ then $T=\left\{\left\langle 0^{m}\right\rangle \mid m \in \omega\right\}$, and $\tilde{T}=F_{\alpha}$. If $\beta>0$ then for almost every $m, \beta_{\sigma(m)}^{\beta}<\beta_{\sigma(m)}^{\alpha}$, and, by Lemma 3, for almost every $m, T(\sigma(m))$ is $\beta_{\sigma(m)}^{\beta}$-dense. Therefore, by induction, for almost every $m$, $\tilde{T}(\sigma(m))$ is $\beta_{\sigma(m)}^{\alpha}$-dense. Thus, again by Lemma $3, \tilde{T}$ is $\alpha$-dense.

The following lemma is the key to the construction. We build trees, again by induction, but with much stronger properties. (However, in the successor ordinal 
case, we lose a finite number of levels, so we can use this construction only for the proof in the limit ordinal case.)

For the sake of convenience, for an arbitrary $\beta<\omega_{1}^{\mathrm{CK}}$ with fixed ordinal notation, define a sequence of predicates $\left\{P_{\alpha}\right\}_{\alpha \leq \beta}$

$$
P_{\alpha}(k) \leftrightarrow \begin{cases}k \in \varnothing^{(\alpha+1)} & \text { if } \alpha \text { is an even ordinal, } \\ k \notin \varnothing^{(\alpha+1)} & \text { otherwise, }\end{cases}
$$

where $\alpha$ is an even ordinal if $\alpha=\lambda+2 n$ for $\lambda \in \operatorname{LOR} \cup\{0\}$ and $n \in \omega$.

LEMMA 6 (STRONG REDUCTION LEMMA). For any recursive ordinal $\alpha$, there exists (uniformly in an ordinal notation for $\alpha$ ) a uniformly recursive sequence $\left\{T_{k}^{\alpha}\right\}_{k \in \omega}$ of trees $T_{k}^{\alpha} \subseteq F_{\alpha}$ such that

$$
\begin{aligned}
P_{\alpha}(k) & \rightarrow\left(\text { a.e. } k_{1}\right)\left(\text { a.e. } k_{2}\right) \ldots\left(\text { a.e. } k_{m}\right)\left[\rho\left(T_{k}^{\alpha}\left(\sigma\left(k_{1}, k_{2}, \ldots, k_{m}\right)\right)\right)<\lambda\right], \text { and } \\
\neg P_{\alpha}(k) & \rightarrow T_{k}^{\alpha} \text { is } \alpha \text {-dense, }
\end{aligned}
$$

where $\alpha=\lambda+m, \lambda \in \mathrm{LOR} \cup\{0\}, m \in \omega$.

PrOOF. For $\alpha=0$, use the construction from (A) above.

For $\alpha$ a successor ordinal, say, $\alpha=\beta+1$, assume without loss of generality that $\alpha$ is even (the odd case is similar). Using $\left(\varnothing^{(\beta+2)}, \overline{\varnothing^{(\beta+2)}}\right) \leq \leq_{1}\left(\operatorname{Fin}^{\varnothing^{(\beta)}}, \operatorname{Cof}^{\varnothing^{(\beta)}}\right)$, there are recursive functions $h$ and $h_{0}$ such that

$$
\begin{aligned}
P_{\alpha}(k) & \rightarrow k \in \varnothing^{(\beta+2)} \rightarrow W_{h_{0}(k)}^{\varnothing^{(\beta)}} \text { finite } \rightarrow\left\{l \mid l \in W_{h_{0}(k)}^{\varnothing^{(\beta)}}\right\} \text { finite } \\
& \rightarrow\left\{l \mid h(k, l) \in \varnothing^{(\beta+1)}\right\} \text { finite } \rightarrow(\text { a.e. } l)\left[P_{\beta}(h(k, l))\right], \\
\neg P_{\alpha}(k) & \rightarrow k \notin \varnothing^{(\beta+2)} \rightarrow W_{h_{0}(k)}^{\varnothing^{(\beta)}} \text { cofinite } \rightarrow\left\{l \mid l \in W_{h_{0}(k)}^{\varnothing^{(\beta)}}\right\} \text { cofinite } \\
& \rightarrow\left\{l \mid h(k, l) \in \varnothing^{(\beta+1)}\right\} \text { cofinite } \rightarrow(\text { a.e. } l)\left[\neg P_{\beta}(h(k, l))\right] .
\end{aligned}
$$

Fix $k$. At stage 0 , put $\varnothing$ into $T_{k, 0}^{\alpha}$. At a stage $s>0$, put $\left\langle 0^{s}\right\rangle$ and $\left\langle 0^{s-1} 1\right\rangle$ into $T_{k, s}^{\alpha}$ and start the construction of $T_{h(k, s-1)}^{\beta}$ on top of $\left\langle 0^{s-1} 1\right\rangle$. The claim that this works is immediate by (25) and Lemma 3 .

For $\alpha$ a limit ordinal, let $\alpha=\left|3 \cdot 5^{x}\right|, \alpha_{n}=\left|\varphi_{x}(n)\right|$, so $\left\{\alpha_{n}\right\}_{n \in \omega}$ is an increasing sequence of ordinals with $\alpha=\sup _{n} \alpha_{n}$. Slightly modify the function $d$ from Lemma 1 so that

$$
(\forall y)\left[y \in \varnothing^{(\alpha+1)} \leftrightarrow(\exists n)\left[P_{\alpha_{n}}(d(y, n))\right]\right]
$$

and, for simplicity,

$$
(\forall n)\left[P_{\alpha_{n}}(d(y, n)) \rightarrow P_{\alpha_{n+1}}(d(y, n+1))\right] .
$$

Given $\sigma \in 2^{<\omega}$, we define the branch number $b(\sigma)=\max \left\{n \mid\left\langle 0^{n}\right\rangle \subseteq \sigma\right\}$, and the decision set $D(\sigma)=\left\{\tau \subseteq \sigma \mid(\exists \tilde{\tau})\left[\tilde{\tau}^{-}\langle 1\rangle=\tau\right]\right\}$. $(b(\sigma)$ will determine the main strategy at $\sigma$, the nodes of $D(\sigma)$ the secondary strategies from lower levels.)

The construction for $\alpha$ a recursive limit ordinal now proceeds as follows: Fix $k$. At stage 0 , put $\varnothing$ into $T_{k, 0}^{\alpha}$. At a stage $s>0$, put $\left\langle 0^{s}\right\rangle$ and $\left\langle 0^{s-1} 1\right\rangle$ into $T_{k, s}^{\alpha}$; also put any $\sigma \in 2^{<\omega}$ into $T_{k, s}^{\alpha}$ for which the following conditions are satisfied:

(i) $|\sigma|=s, \sigma \uparrow(s-1) \in T_{k, s-1}^{\alpha}$,

(ii) $\sigma \in F_{\alpha}$, and

(iii) $(\forall \tau \in D(\sigma))(\forall m \leq b(\sigma))\left[\alpha_{m} \leq \beta_{\tau}^{\alpha} \wedge \sigma \in \tau^{\wedge} F_{\alpha_{m}} \rightarrow \sigma \in \tau^{\wedge} T_{d(k, m)}^{\alpha_{m}}\right]$. 
(Notice here that the construction is arranged in such a way that to any $\sigma\left(k_{1}, k_{2}, \ldots, k_{m}\right)$, the construction above it looks the same as to a $\sigma(n)$ above it. This will be an essential feature for the verification.)

Now suppose first that $k \in \varnothing^{(\alpha+1)}$, i.e., by the modification of Lemma 1 , $P_{\alpha_{n}}(d(k, n))$ holds for all $n \geq$ some fixed $n_{0}$. We then claim that $\rho\left(T_{k}^{\alpha}(\sigma(n))\right) \leq \alpha_{n_{0}}$ for all $n$, thus $\rho\left(T_{k}^{\alpha}\right) \leq \alpha_{n_{0}}+1<\alpha$ as desired. The proof requires induction on $\alpha_{n_{0}}$. (Of course, there is nothing to prove for $\alpha_{n} \leq \alpha_{n_{0}}$.)

$\alpha_{n_{0}}=0$ : Let $\tilde{\tau}=\sigma(n)$. Then $\tilde{\tau}^{\wedge} F_{\alpha_{n_{0}}}=\left\{\sigma(n)^{\wedge}\left\langle 0^{m}\right\rangle \mid m \in \omega\right\}$, so $\left\langle 0^{m_{0}}\right\rangle \notin$ $T_{d\left(k, n_{0}\right)}^{\alpha_{n_{0}}}$ for some $m_{0}$, and thus $T_{k}^{\alpha}\left(\tilde{\tau}^{\wedge}\left\langle 0^{m_{0}}\right\rangle\right)$ is finite. As for $T_{k}^{\alpha}(\sigma(n, m))$ for $m<m_{0}$, apply the same proof to $\tilde{\tau}=\sigma(n, m)$, etc. By Lemma 2, there is no infinite sequence $\sigma(n), \sigma(n, m), \sigma(n, m, l), \ldots$ of such $\tilde{\tau}$ 's, so $T_{k}^{\alpha}(\sigma(n))$ is finite and $\rho\left(T_{k}^{\alpha}(\sigma(n))\right) \leq \alpha_{n_{0}}$.

$\alpha_{n_{0}}=\beta+1$ : There is $m_{0}$ such that $P_{\beta}\left(h\left(d\left(k, n_{0}\right), m\right)\right)$ holds for all $m \geq m_{0}$ where $h$ is the function for $\alpha_{n_{0}}$ and $\beta$ mentioned above in the proof for the successor ordinal case. Now the $\alpha_{n_{0}}$-construction works at $\sigma(n)$, and thus the $\beta$-construction at $\sigma(n, m)$ for all $m$, through condition (iii) of the construction (putting $\tau=\sigma(n)$ ). Thus by induction (replacing $\alpha_{n_{0}}$ and $\alpha_{n}$ by $\beta$ and $\beta_{\sigma(m)}^{\alpha_{n}}$ ), there is some $m_{0}$ such that $\rho\left(T_{k}^{\alpha}(\sigma(n, m))\right) \leq \beta$ for all $m \geq m_{0}$, so $\rho\left(T_{k}^{\alpha}\left(\sigma(n)^{\wedge}\left\langle 0^{m_{0}}\right\rangle\right)\right) \leq \alpha_{n_{0}}$. As for $T_{k}^{\alpha}(\sigma(n, m))$ for $m<m_{0}$, apply the same proof with $\tau=\sigma(n, m)$, etc. By Lemma 2 , there is no infinite sequence $\sigma(n), \sigma(n, m), \sigma(n, m, l), \ldots$ of such $\tau$ 's, so $T_{k}^{\alpha}(\sigma(n))$ consists of finitely many subtrees, each of rank $\leq \alpha_{n_{0}}$, and thus $\rho\left(T_{k}^{\alpha}(\sigma(n))\right) \leq \alpha_{n_{0}}$.

The above establishes $\rho\left(T_{k}^{\alpha}(\sigma(n))\right) \leq \alpha_{n_{0}}<\alpha$ for all $n$, so $\rho\left(T_{k}^{\alpha}\right) \leq \alpha_{n_{0}}+1<\alpha$ in the successor ordinal case of $\alpha_{n_{0}}$.

$\alpha_{n_{0}} \in$ LOR: Then $\left\{\beta_{\sigma(m)}^{\alpha_{n_{0}}}\right\}_{m \in \omega}$ is an increasing sequence with limit $\alpha_{n_{0}}$. There is $m_{0}$ such that $P_{\beta_{\sigma(m)}^{\alpha_{n}}}\left(\tilde{d}\left(d\left(k, n_{0}\right), m\right)\right)$ holds for all $m \geq m_{0}$ where $\tilde{d}$ is the counterpart of $f$ for $\alpha_{n_{0}}$ as a limit ordinal. Now the $\alpha_{n_{0}}$-construction works at $\sigma(n)$, and thus the $\beta_{\sigma(m)}^{\alpha_{n_{0}}}$-construction at $\sigma(n, m)$ for all $m$, through condition (iii) of the construction (putting $\tau=\sigma(n)$ ). Thus by induction (replacing $\alpha_{n_{0}}$ and $\alpha_{n}$ by $\beta_{\sigma(m)}^{\alpha_{n_{0}}}$ and $\left.\beta_{\sigma(m)}^{\alpha_{n}}\right)$, we have that $\rho\left(T_{k}^{\alpha}(\sigma(n, m))\right) \leq \beta_{\sigma\left(m_{0}\right)}^{\alpha_{n_{0}}}$ for all $m \geq m_{0}$ (this part does not follow by induction for $m$ with $\beta_{\sigma(m)}^{\alpha_{n}} \leq \beta_{\sigma(m)}^{\alpha_{n_{0}}}$ but in that case it is trivial anyway). Therefore, $\rho\left(T_{k}^{\alpha}\left(\sigma(n) \wedge\left\langle 0^{m_{0}}\right\rangle\right)\right) \leq \alpha_{n_{0}}$. As for $T_{k}^{\alpha}(\sigma(n, m))$ for $m<m_{0}$, apply the same proof with $\tau=\sigma(n, m)$, etc. By Lemma 2, there is no infinite sequence $\sigma(n), \sigma(n, m), \sigma(n, m, l), \ldots$ of such $\tau$ 's, so $T_{k}^{\alpha}(\sigma(n))$ consists of finitely many subtrees, each of rank $\leq \alpha_{n_{0}}$, so $\rho\left(T_{k}^{\alpha}(\sigma(n))\right) \leq \alpha_{n_{0}}$.

The above establishes $\rho\left(T_{k}^{\alpha}(\sigma(n))\right) \leq \alpha_{n_{0}}<\alpha$ for all $n$, so $\rho\left(T_{k}^{\alpha}\right) \leq \alpha_{n_{0}}+1<\alpha$ in the limit ordinal case of $\alpha_{n_{0}}$.

On the other hand, assume that $k \notin \varnothing^{(\alpha+1)}$. Then $P_{\alpha_{n}}(d(k, n))$ does not hold for any $n$. We claim that $T_{k}^{\alpha}$ is $\alpha$-dense (and thus $\left[D^{\alpha}\left(T_{k}^{\alpha}\right)\right]=\left\{\left\langle 0^{\omega}\right\rangle\right\}$ ). We proceed by induction on $\beta=\alpha_{n}$, using Lemma 3:

$\alpha_{n}=0:$ We have $T_{k}^{\alpha}(\sigma(n))=T_{d(k, n)}^{0}=\left\{\left\langle 0^{m}\right\rangle \mid m \in \omega\right\}$, so $\rho(T(\sigma(n)))=\alpha_{n}$.

$\alpha_{n}>0$ : We have

$$
\begin{aligned}
T_{k}^{\alpha}(\sigma(n))=\left\{\sigma \in F_{\alpha_{n}} \mid(\forall \tilde{\sigma} \subseteq \sigma)(\forall \tau \in D(\tilde{\sigma}) \cup\{\varnothing\})(\forall m \leq n)\right. \\
{\left.\left[\alpha_{m} \leq \beta_{\tau}^{\alpha_{n}} \wedge \tilde{\sigma} \in \tau^{\wedge} F_{\alpha_{m}} \rightarrow \tilde{\sigma} \in \tau^{\wedge} T_{d(k, m)}^{\alpha_{m}}\right]\right\} }
\end{aligned}
$$


Among these restrictions, we can distinguish three types:

(a) $\tau \neq \varnothing$ (and thus $m<n)$;

(b) $\tau=\varnothing$ and $m=n$; and

(c) $\tau=\varnothing$ and $m<n$.

Thus $T(\sigma(n))$ is the intersection of the following three trees:

(a) $T_{1}=\left\{\sigma \in F_{\alpha_{n}} \mid(\forall \tilde{\sigma} \subseteq \sigma)(\forall \tau \in D(\tilde{\sigma}))(\forall m<n)\left[\alpha_{m} \leq \beta_{\tau}^{\alpha_{n}} \wedge \hat{\sigma} \in\right.\right.$ $\left.\left.\tau^{\wedge} F_{\alpha_{m}} \rightarrow \tilde{\sigma} \in \tau^{\wedge} T_{d(k, m)}^{\alpha_{m}}\right]\right\}$

(b) $T_{2}=\left\{\sigma \in F_{\alpha_{n}} \mid(\forall \tilde{\sigma} \subseteq \sigma)\left[\tilde{\sigma} \in T_{d(k, n)}^{\alpha_{n}}\right]\right\}=T_{d(k, n)}^{\alpha_{n}}$; and

(c) $T_{3}=\left\{\sigma \in F_{\alpha_{n}} \mid(\forall \tilde{\sigma} \subseteq \sigma)(\forall m<n)\left[\tilde{\sigma} \in F_{\alpha_{m}} \rightarrow \tilde{\sigma} \in T_{d(k, m)}^{\alpha_{m}}\right]\right\}=$ $\bigcap_{m<n}\left\{\sigma \in F_{\alpha_{n}} \mid(\forall \tau \subseteq \sigma)\left[\tau \in F_{\alpha_{m}} \rightarrow \tau \in T_{d(k, m)}^{\alpha_{m}}\right]\right\}$. (Call these trees $T_{3, m}$ for $m<n$.)

By Lemma 4, it suffices to show that each of $T_{1}, T_{2}$, and the $T_{3, m}$ is $\alpha_{n}$-dense.

(a) Recall again the remark that the construction above $\sigma(n)$ looks to $\alpha_{n}$ just as it does to $\alpha$ above $\varnothing$. For all $l$,

$$
\begin{array}{r}
T_{1}(\sigma(l))=\left\{\sigma \in F_{\beta_{\sigma(l)}^{\alpha_{n}}} \mid(\forall \tilde{\sigma} \subseteq \sigma)(\forall \tau \in D(\tilde{\sigma}) \cup\{\varnothing\})(\forall m<n)\right. \\
\left.\left[\alpha_{m} \leq \beta_{\sigma(l) \uparrow \tau}^{\alpha_{n}} \wedge \tilde{\sigma} \in \tau^{-} F_{\alpha_{m}} \rightarrow \tilde{\sigma} \in \tau^{\wedge} T_{d(k, m)}^{\alpha_{m}}\right]\right\} .
\end{array}
$$

Therefore, by induction on $\beta=\alpha_{n}$ in (28) (with $\beta_{\sigma(l)}^{\alpha_{n}}$ in place of $\alpha_{n}$, and $\beta_{\sigma(l) \tau}^{\alpha_{n}}$ in place of $\left.\beta_{\tau}^{\alpha_{n}}\right), T_{1}(\sigma(l))$ is $\beta_{\sigma(l)}^{\alpha_{n}}$-dense for almost every $l$. Thus, by Lemma $3, T_{1}$ is $\alpha_{n}$-dense.);

(b) $T_{2}$ is $\alpha_{n}$-dense by induction on the overall construction; and

(c) each $T_{3, m}$ is $\alpha_{n}$-dense by induction and Lemma 5 .

This concludes the proof of Lemma 6 .

Lemma 6 now implies part (D) of the proof of the Reduction Lemma, and thus Theorem $\mathrm{B}$ has been established.

6. A $\Pi_{1}^{1}$-complete index set. We can extend the above techniques to obtain a $\Pi_{1}^{1}$-complete index set. (This follows up a suggestion by J. Steel.) We will use the fact that Kleene's set $O$ of ordinal notations is a $\Pi_{1}^{1}$-complete subset of $\omega$.

Call a map $f$ from a partially ordered set (poset) $X$ into a poset $Y$ an order embedding if

$$
(\forall x, y \in X)\left[x \leq_{X} y \leftrightarrow f(x) \leq_{Y} f(y)\right] .
$$

(In particular, any order embedding is a 1-1 map.) Then we define:

DEFinition. Let $A \subseteq \omega$ be a hyperhypersimple or cofinite set. Then $A$ is $B$-atomic if there is no order embedding $f$ from the countable atomless Boolean algebra $B$ into $\mathcal{L}^{*}(A)$, the lattice of r.e. supersets of $A$ (modulo finite sets).

The following equivalent definition is easier to handle:

Proposition. Let $A \subseteq \omega$ be a hyperhypersimple or cofinite set. Then $A$ is $B$-atomic iff $A$ is $\alpha$-atomic for some ordinal $\alpha$.

PROOF. We will represent the countable atomless Boolean algebra $B$ by certain subsets of $2^{<\omega}$.

Given $\sigma \in 2^{<\omega}$, let $U_{\sigma}=\left\{\tau \in 2^{<\omega} \mid \sigma \subseteq \tau\right\}$ be the "cone" above $\sigma$. Let $T \subseteq 2^{<\omega}$ be a tree. Call $U \subseteq T$ a finite regular cut in $T$ if:

(i) $U$ is of the form $U=T \cap \bigcup_{\sigma \in S} U_{\sigma}$ for some finite (possibly empty) set $S \subseteq T ;$ and 
(ii) for any $\sigma \in T-U$, there is some $\tau \supset \sigma$ such that $\tau \in T-U$.

Then the set of finite regular cuts in $T$ forms a Boolean algebra under the following operations (see, e.g., Jech [Je78], for a similar construction):

(meet)

$$
\begin{aligned}
U \vee V & =\text { least finite regular cut containing } U \cup V \\
& =\left\{\sigma \in T \mid(\forall \tau \in T)\left[\sigma \subseteq \tau \rightarrow(U \cup V) \cap U_{\tau} \neq \varnothing\right]\right\},
\end{aligned}
$$

(complement)

$$
U \wedge V=U \cap V
$$

(ordering)

$-U=$ greatest finite regular cut disjoint from $U$

$$
=\left\{\sigma \in T \mid U \cap U_{\sigma}=\varnothing\right\},
$$

Let $B$ be the set of finite regular cuts in $2^{<\omega}$. Then $B$ is the countable atomless Boolean algebra (since for any $\left.\sigma \in 2^{<\omega}, U_{\sigma} \supset U_{\sigma<\langle 0\rangle} \supset \varnothing\right)$.

First assume that $A$ is not $\alpha$-atomic for any ordinal $\alpha$. Then define the following increasing sequence of filters in $\mathcal{L}^{*}(A)$. Let $\mathcal{F}_{0}$ be the one-element filter, let $\mathcal{F}_{\alpha+1}$ be the filter in $\mathcal{L}^{*}(A)$ generated by the coatoms in $\mathcal{L}^{*}(A) / \mathcal{F}_{\alpha}$, and let $\mathcal{F}_{\lambda}=\bigcup_{\alpha<\lambda} \mathcal{F}_{\alpha}$ for $\lambda$ a limit ordinal. Since $A$ is not $\alpha$-atomic for any $\alpha, \mathcal{L}^{*}(A) / \mathcal{F}_{\alpha}$ is not finite for any ordinal $\alpha$. Since $\mathcal{L}^{*}(A)$ is countable, $\mathcal{F}_{\alpha_{0}}=\mathcal{F}_{\alpha_{0}+1}$ for some $\alpha_{0}<\omega_{1}$. Therefore, $\mathcal{L}^{*}(A) / \mathcal{F}_{\alpha_{0}}$ is the countable atomless Boolean algebra. Fix an isomorphism $i$ from $B$ into $\mathcal{L}^{*}(A) / \mathcal{F}_{\alpha_{0}}$. The idea is now to "lift" $i$ to an order embedding $f$ from $B$ into $\mathcal{L}^{*}(A)$. Let $h$ be a map from $\mathcal{L}^{*}(A) / \mathcal{F}_{\alpha_{0}}$ into $\mathcal{L}^{*}(A)$, picking a representative for each equivalence class. Now define

$$
\begin{aligned}
f(\varnothing) & =A^{*}, \\
f\left(U_{\varnothing}\right) & =h\left(i\left(U_{\varnothing}\right)\right), \\
f\left(U_{\sigma^{\curlyvee}\langle k\rangle}\right) & =f\left(U_{\sigma}\right) \cap h\left(i\left(U_{\sigma^{\prec}\langle k\rangle}\right)\right) \text { for } \sigma \in 2^{<\omega}, k \in 2 .
\end{aligned}
$$

This obviously induces an order homomorphism from $B$ into $\mathcal{L}^{*}(A)$. It remains to check that $f$ is $1-1$. But $i$ is $1-1$, and $h(U)=f(U)\left(\bmod \mathcal{F}_{\alpha_{0}}\right)$; therefore $f$ has to be 1-1 also.

Conversely, assume that there is a hyperhypersimple set $A$ which is $\alpha$-atomic for some $\alpha$ but not B-atomic. Pick $\alpha_{0}$ to be the minimal such ordinal. We will reach a contradiction by showing that $\alpha_{0}$ cannot be minimal.

Let $A$ be $\alpha_{0}$-atomic but not B-atomic. Let $g$ be an order embedding from $B$ into $\mathcal{L}^{*}(A)$. If $g\left(U_{\langle 0\rangle}\right)$ is in the $<\alpha_{0}$-atomic filter $\mathcal{F}$ then put $A_{0}=g\left(U_{\langle 0\rangle}\right)$, and $f \uparrow\left\{U \in B \mid U_{\langle 0\rangle} \subseteq U\right\}$ is an order embedding of a countable atomless Boolean algebra into $\mathcal{L}^{*}\left(A_{0}\right)$. Otherwise, since $A$ is $\alpha_{0}$-atomic (and thus $\left|\mathcal{L}^{*}\left(A_{0}\right) / \mathcal{F}\right| \leq 2$ ), we have that $A=g\left(U_{\langle 0\rangle}\right)(\bmod \mathcal{F})$. So $A_{0}=A \cup \overline{g\left(U_{\langle 0\rangle}\right)}$ is $<\alpha_{0}$-atomic, and the map $\left\{U \in B \mid U \subseteq U_{\langle 0\rangle}\right\} \rightarrow \mathcal{L}^{*}\left(A_{0}\right), U \mapsto A \cup \overline{g(U)}$ is an order embedding of a countable atomless Boolean algebra into $\mathcal{L}^{*}\left(A_{0}\right)$.

In either case, we have a $<\alpha_{0}$-atomic (say, a $\beta_{0}$-quasiatomic) set $A_{0}$ and an order embedding $f$ from $B$ into $\mathcal{L}^{*}\left(A_{0}\right)$. Let $\mathcal{F}_{0}$ be the $<\beta_{0}$-quasiatomic filter in $\mathcal{L}^{*}\left(A_{0}\right)$. Then $\mathcal{L}^{*}\left(A_{0}\right) / \mathcal{F}_{0}$ is finite since $A_{0}$ is $\beta_{0}$-quasiatomic. If $\left|\mathcal{L}^{*}\left(A_{0}\right) / \mathcal{F}_{0}\right| \leq 2$ then $A_{0}$ is $\beta_{0}$-atomic, and we have already reached a contradiction. Otherwise, pick two sets $A_{1} \subset A_{2}$, satisfying the following conditions:

(i) there are $U_{1}, U_{2} \in B$ such that $f\left(U_{1}\right)=A_{1}^{*}, f\left(U_{2}\right)=A_{2}^{*}$, and $U_{1} \subset U_{2}$; 
(ii) $B_{0}=\left\{U \in B \mid U_{1} \subseteq U \subseteq U_{2}\right\}$ forms a countable atomless Boolean algebra; and

(iii) $A_{1}^{*}$ and $A_{2}^{*}$ represent distinct equivalence classes $c_{1}$ and $c_{2}$ in $\mathcal{L}^{*}\left(A_{0}\right) / \mathcal{F}_{0}$ such that there is no $c \in \mathcal{L}^{*}\left(A_{0}\right) / \mathcal{F}_{0}$ with $c_{1}<c<c_{2}$.

Let $h$ be the map $W \mapsto W \cup \overline{A_{2}}$. Then $h \circ\left(f \uparrow\left\{W^{*} \mid A_{1} \subseteq W \subseteq A_{2}\right.\right.$ r.e. $\left.\}\right)$ is an order embedding from $B_{0}$ into $\mathcal{L}^{*}\left(A_{1} \cup \overline{A_{2}}\right)$, and $A_{1} \cup \overline{A_{2}}$ is $\beta_{0}$-atomic, a contradiction.

Let $A t_{B}$ be the index set of B-atomic sets. We are now in a position to exhibit a $\Pi_{1}^{1}$-complete index set. Recall that Kleene's $O$, the set of ordinal notations, is $\Pi_{1}^{1}$-complete.

THEOREM C. $0 \leq_{1} \mathrm{At}_{\mathrm{B}}$.

COROLlary. The index set of B-atomic sets is $\Pi_{1}^{1}$-complete.

Proof. It suffices to show that $\mathrm{At}_{\mathrm{B}}$ is $\Pi_{1}^{1}$. But

$x \in \mathrm{At}_{\mathrm{B}} \leftrightarrow \neg(\exists f: B \rightarrow \omega)(\forall U, V \in B)\left[W_{x} \subseteq W_{f(U)} \wedge\left(U \leq V \leftrightarrow W_{f(U)} \subseteq^{*} W_{f(V)}\right)\right]$.

(Of course, $B$ can easily be coded by the integers.)

PROOF OF THEOREM C. Extending our machinery to $\Pi_{1}^{1}$, we again have to prove a Correspondence Lemma and a Reduction Lemma.

CORRESPONDENCE LemMa. Let $T \subseteq 2^{<\omega}$ be a $\Sigma_{2}$-tree. Then $\rho(T)<\infty$ iff $A_{T}$ is B-atomic.

PROOF. By the Correspondence Lemma for Theorem B and the above proposition, $\rho(T)<\infty$ iff $(\exists \alpha)[\rho(T) \leq \alpha]$ iff $(\exists \alpha)\left[A_{T}\right.$ is $(1+\alpha)$-quasiatomic $]$ iff $A_{T}$ is B-atomic.

Now let $\tau=\left\{T \subseteq 2^{<\omega} \mid \rho(T)<\infty\right\}$.

REDUCTION LEMMA. There is a 1-1 reduction $0 \leq_{1}$ indices of recursive trees of $\tau$.

PROOF. Let us first analyze how we can "discover" that some integer $x$ is not an ordinal notation. We will illustrate this with the help of a partial map $S_{x}$ (predecessor tree) from $\omega^{<\omega}$ to $\omega$. Define $S_{x}: \omega^{<\omega} \mapsto \omega$ by induction as follows:

$$
\begin{aligned}
S_{x}(\varnothing) & =x, \\
S_{x}\left(\sigma^{\wedge}\langle i\rangle\right) & = \begin{cases}y & \text { if } S_{x}(\sigma)=2^{y} \text { and } y \neq 0, \\
\{z\}(i) & \text { if } S_{x}(\sigma)=3 \cdot 5^{z}, \\
\text { undefined } & \text { otherwise. }\end{cases}
\end{aligned}
$$

Let $P_{x}$ (set of predecessor notations of $x$ ) be the set $P_{x}=\left\{S_{x}(\sigma) \downarrow \mid \sigma \in \omega^{<\omega}-\right.$ $\{\varnothing\}\}$.

Then $x$ is not an ordinal notation iff one of the following holds:

(i) $S_{x}$ contains an infinite path (i.e., there is an infinite descending chain of predecessors of $x$ );

(ii) for some $\sigma \in \omega^{<\omega}$ and some $i, S_{x}(\sigma) \downarrow \neq 1$ and $S_{x}\left(\sigma^{\wedge}\langle i\rangle\right) \uparrow$; or

(iii) for some $\sigma \in \omega^{<\omega}$ and some $i<j, S_{x}(\sigma)$ is of the form $3 \cdot 5^{z}$ and $S_{x}\left(\sigma^{\wedge}\langle i\rangle\right) \downarrow$ $\notin P_{S_{x}(\widehat{\sigma}\langle j\rangle)}$. 
Now, given $x$, construct a recursive tree $T_{x}$ as follows. The tree will be recursive by induction on the length of strings $\sigma \in 2^{<\omega}$. Define

$$
T_{x}= \begin{cases}\left\{\left\langle 0^{i}\right\rangle \mid i \in \omega\right\} & \text { if } x=1, \\ T_{1} \cup \bigcup_{i \in \omega}\left(\left\langle 0^{i} 10\right\rangle^{\wedge} T_{y}\right) \cup \bigcup_{i \in \omega}\left(\left\langle 0^{i} 1^{2}\right\rangle^{\wedge} T_{y}\right) & \text { if } x=2^{y} \text { and } y \neq 0, \\ T_{1} \cup \bigcup_{i \in \omega}\left(\left\langle 0^{i} 10\right\rangle^{\wedge} \tilde{T}_{z, i}\right) \cup \bigcup_{i \in \omega}\left(\left\langle 0^{i} 1^{2}\right\rangle^{\wedge} \tilde{T}_{z, i}\right) & \text { if } x=3 \cdot 5^{z}, \\ 2^{<\omega} & \text { otherwise. }\end{cases}
$$

Here, $\tilde{T}_{z, i}$ is defined as follows:

(35)

$$
\tilde{T}_{z, i}=\left\{\begin{array}{lc}
2^{<s} \cup\left(\left\langle 0^{s}\right\rangle^{\wedge} T_{\{z\}(i)}\right) & \text { if }\{z\}_{s}(i) \downarrow \text { and, if } i \neq 0, \\
& \{z\}_{s}(i-1) \downarrow \in P_{\{z\}(i), s} \\
2^{<\omega} & \text { otherwise. }
\end{array}\right.
$$

First suppose that $x$ is an ordinal notation. We prove by induction on $|x|$ that $\rho\left(T_{x}\right)=|x|<\infty$. There are three cases:

$x=1$ : Then $\left|\left[T_{x}\right]\right|=1$ and $\rho\left(T_{x}\right)=0<\infty$.

$x=2^{y}$ : Then $\rho\left(T_{x}\right)=\rho\left(T_{y}\right)+1=|y|+1=|x|<\infty$.

$x=3 \cdot 5^{z}$ : Then $\rho\left(\tilde{T}_{z, i}\right)=|\{z\}(i)|$ and therefore $\rho\left(T_{x}\right)=\sup _{i \in \omega}|\{z\}(i)|=|x|$.

On the other hand, assume that $x$ is not an ordinal notation. Then one of the above conditions (i)-(iii) holds:

(i) There is an infinite descending chain of predecessors of $x$, say, $x=x_{0}, x_{1}, x_{2}$, $x_{3}, \ldots$. Then, by construction, there is a sequence $\sigma_{1}, \sigma_{2}, \sigma_{3}, \ldots$ of binary strings such that, for all $i>0$,



for any $i$-tuple $\left(k_{1}, k_{2}, \ldots, k_{i}\right) \in 2^{i}$. Therefore

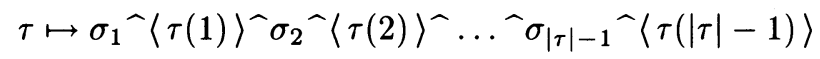

is a mapping from $2^{<\omega}$ into a perfect subtree of $T_{x}$, and so $\rho\left(T_{x}\right)=\infty$.

(ii) For some $\sigma \in \omega^{<\omega}$ and some $i \in \omega, S_{x}(\sigma) \downarrow \neq 1$ and $S_{x}\left(\sigma^{\wedge}\langle i\rangle\right) \uparrow$. Then $\tau^{\wedge} T_{S_{x}(\sigma)} \subseteq T_{x}$ for some $\tau \in 2^{<\omega}$; and for the construction of $T_{S_{x}(\sigma)}$, the second clause of (35) or the fourth clause of (34) applies. Therefore, $\tau^{\wedge}\left\langle 0^{i} 10\right\rangle^{\wedge} 2^{<\omega} \subseteq T_{x}$, and so $\rho\left(T_{x}\right)=\infty$.

(iii) For some $\sigma \in \omega^{<\omega}$ and some $i<j, S_{x}(\sigma)=3 \cdot 5^{z}$ and $S_{x}\left(\sigma^{\wedge}\langle i\rangle\right) \downarrow \notin$ $P_{S_{x}\left(\sigma^{\wedge}\langle j\rangle\right)}$. Then again $\tau^{\wedge} T_{S_{x}(\sigma)} \subseteq T_{x}$ for some $\tau \in 2^{<\omega}$, and the second clause of (35) applies for $\tilde{T}_{z, j}$. Therefore, $\tau^{\wedge}\left\langle 0^{j} 10\right\rangle^{\wedge} 2^{<\omega} \subseteq T_{x}$, and so $\rho\left(T_{x}\right)=\infty$.

This concludes the proof of Theorem C.

7. An index set in major subsets. Lachlan [La68] defined the following notion of two r.e. sets $A \subset_{\infty} B$ being "close" to each other:

Definition. Let $A \subset_{\infty} B$ be r.e. sets. Then $A$ is major in $B\left(A \subset_{\mathrm{m}} B\right)$ iff

$$
(\forall W \text { r.e. })\left[\bar{B} \subseteq \subseteq^{*} W \rightarrow \bar{A} \subseteq \subseteq^{*} W\right]
$$

(38) is equivalent to either of the following two conditions:

$$
\begin{gathered}
(\forall W \text { r.e. })\left[\bar{B} \subseteq W \rightarrow \bar{A} \subseteq \subseteq^{*} W\right], \\
\mathcal{L}^{*}(\bar{A})=\mathcal{L}^{*}(\bar{B}),
\end{gathered}
$$

where $\mathcal{L}^{*}(X)$ is the lattice of r.e. supersets of $X$ (modulo finite sets). 
The classification of the index set $\left\{\langle e, i\rangle \mid W_{e} \subset_{\mathrm{m}} W_{i}\right\}$ has been one of the open questions in index sets for a while. The major obstacle here is that $A \subset_{\mathrm{m}} B$ implies that $B$ is nonrecursive. This makes the uniformity required for the classification hard. We present below a partial result towards the classification of this index set:

THEOREM D. Let $V$ be a nonrecursive r.e. set. Then the index set $\mathrm{Maj}_{V}=$ $\left\{k \mid W_{k} \subset_{\mathrm{m}} V\right\}$ is $\Pi_{4}$-complete.

PROOF. It is easy to see that $\mathrm{Maj}_{V}$ is $\Pi_{4}$ :

$$
\begin{aligned}
W_{k} \subset_{\mathrm{m}} V & \leftrightarrow W_{k} \subset_{\infty} V \wedge(\forall e)\left[V \cup W_{e} \neq \omega \vee W_{k} \cup W_{e}={ }^{*} \omega\right] \\
& \leftrightarrow \Pi_{3} \wedge(\forall e)\left[\Sigma_{2} \vee \Sigma_{3}\right] \\
& \leftrightarrow \Pi_{4} .
\end{aligned}
$$

We will build (uniformly in $k$ ) an r.e. set $A_{k} \subset_{\infty} V$ such that $A_{k} \subset_{\mathrm{m}} V$ iff $k \notin \varnothing^{(4)}$. (We will usually suppress the index $k$ on $A$ from now on.)

We use the fact that there is a recursive function $h$ such that

$$
\begin{aligned}
& k \notin \varnothing^{(4)} \rightarrow(\forall i)\left[W_{h(k, i)} \text { cofinite }\right], \\
& k \in \varnothing^{(4)} \rightarrow(\exists i)\left[W_{h(k, i)} \text { coinfinite }\right] .
\end{aligned}
$$

Fix $k$ from now on, and let $\overline{W_{h(k, i), s}}=\left\{h_{i, 0}^{s}<h_{i, 1}^{s}<h_{i, 2}^{s}<\cdots\right\}$.

The idea of the proof is now to have for each $i$ two conflicting strategies, a positive strategy trying to establish $\left(38^{\prime}\right)$ for $W_{i}$, and a negative strategy trying to build a counterexample $B$ to $A \subset_{\mathrm{m}} V$. Which strategy succeeds will depend on whether $W_{h(k, i)}$ is cofinite or not. (If $W_{h(k, i)}$ is coinfinite then the strategies working on $i^{\prime}>i$ will not matter.)

For the basic module of the positive $P_{e}$-strategy, we use a variant of Lachlan's strategy [La68] to construct a major subset. Let $\tilde{W}_{e, s}=\left\{x \in W_{e, s} \mid(\forall y<x)[y \in\right.$ $\left.\left.W_{e, s} \cup V_{s}\right]\right\}$, and let $\tilde{W}_{e}=\cup_{s} \tilde{W}_{e, s}$. Then $W_{e}=\tilde{W}_{e}$ if $W_{e} \supseteq \bar{V}$, and $\tilde{W}_{e}$ is finite if $W_{e} \nsupseteq \bar{V}$. In the former case, we have to take action for the sake of $W_{e}$; in the latter case, the strategy will only have a finite effect on the rest of the construction. Furthermore, let $f$ be a 1-1 enumeration of $V$ (recall that $V$ has to be infinite). Finally, let $V_{s}-A_{s}=\left\{d_{0}^{s}, d_{1}^{s}, d_{2}^{s}, \ldots, d_{n_{s}}^{s}\right\}$ where the markers $d_{n}^{s}$ need not be in order. (The markers $d_{n}^{s}$ will be undefined for $n>n_{s}$.)

At stage 0 , let $A_{0}=\varnothing$, let $d_{0}^{0}=f(0)$, and let $d_{n}^{0}$ be undefined for $n>0$. At a stage $s+1$, first determine if $f(s+1) \in \tilde{W}_{e, s}$ and $d_{\tilde{n}}^{s} \notin \tilde{W}_{e, s}$ for some $\tilde{n} \leq n_{s}$. If so, for the least such $\tilde{n}$, put $d_{\tilde{n}}^{s}$ into $A_{s+1}$, let $d_{\tilde{n}}^{s+1}=f(s+1)$, and let $d_{n}^{s+1}=d_{n}^{s}$ for all $n \neq \tilde{n}$ (for the sake of $\bar{A} \subseteq{ }^{*} W_{e}$ ). Otherwise, let $d_{n_{s}+1}^{s+1}=f(s+1)$, and let $d_{n}^{s+1}=d_{n}^{s}$ for $n \neq n_{s}+1$ (for the sake of $A \subset_{\infty} V$ ).

Since $V$ is nonrecursive, $\bar{V}$ is not r.e. Suppose $\bar{V} \subseteq W_{e}$ (and thus $W_{e}=\tilde{W}_{e}$ ). Since $\left\{x \mid(\exists s)\left[x \in \tilde{W}_{e, s} \wedge x \in \bar{V}_{s}\right]\right\}$ is r.e. and contains $\bar{V}$ we have that

$$
\left(\exists^{\infty} s\right)(\exists x)\left[x \in V_{s+1}-V_{s} \wedge x \in \tilde{W}_{e, s}\right] \text {. }
$$

Therefore, $f(s+1) \in \tilde{W}_{e, s}$ for infinitely many $s$, so any marker $d_{n}^{s}$ will be moved until it is in $\tilde{W}_{e}$, and so $\bar{A} \subseteq \tilde{W}_{e}$. (These strategies will later be combined using $e$-states as first introduced by Friedberg in his maximal set construction [Fr58].)

The basic module for the negative $\mathcal{N}$-strategy tries to build a set $B$ refuting $A \subset_{\mathrm{m}} V$, i.e., such that $\bar{V} \subseteq B$ and that $V-(A \cup B)$ is infinite. At the $n$th time 
the strategy acts, it will wait for $|V-(A \cup B)|>n$, then put $\min (\bar{V})$ into $B$ (for the sake of $\bar{V} \subseteq B)$ and restrain another element of $V-(A \cup B)$ from entering $A$ (to make $V-(A \cup B)$ infinite).

Suppose that $A \subset_{\infty} V$. Then the strategy will act infinitely often (else $B$ and thus $V-A$ would be finite). So $\bar{V} \subseteq B$ and $V-(A \cup B)$ is infinite. (Notice that we really only have to restrain forever from $A$ an infinite subset of the restrained elements of $V-(A \cup B)$.)

We have to let the success (or failure) of the $\mathcal{N}$-strategy depend on whether $W_{h(k, i)}$ is coinfinite (or cofinite). Recall that $\overline{W_{h(k, i), s}}=\left\{h_{i, 0}^{s}<h_{i, 1}^{s}<h_{i, 2}^{s}<\cdots\right\}$. Let the $\mathcal{N}$-strategy only restrain at stage $s+1$ at most $m_{s}=\min \left\{n \mid h_{i, s+1}^{n} \neq h_{i, s}^{n}\right\}$ many elements. If $W_{h(k, i)}$ is coinfinite then $\lim _{s} m_{s}=\infty$, so the $\mathcal{N}$-strategy can eventually restrain more and more elements from $A$ permanently. If $W_{h(k, i)}$ is cofinite then $m=\liminf _{s} m_{s}<\infty$, so the $\mathcal{N}$-strategy can restrain at most $m$ elements permanently from $A$. (Notice that if one $\mathcal{N}$-strategy is allowed to succeed the lower-priority $\mathcal{P}$-strategies will not matter since this $\mathcal{N}$-strategy will satisfy the overall requirement $A \not \subset_{\mathrm{m}} V$.)

Combining all strategies requires two minor changes:

First of all, a stronger $\mathcal{P}$-strategy may injure a weaker $\mathcal{N}$-strategy by putting infinitely many elements into $A$ that are restrained by the $\mathcal{N}$-strategy. So the latter has to be able to predict which elements the $P$-strategy will put into $A$. This is done in a straightforward tree argument fashion.

Secondly, if a $P$-strategy is forced to always observe the current restraint of the stronger $\mathcal{N}$-strategies then a synchronization problem may arise. Good elements (i.e., numbers $\left.f(s+1) \in \tilde{W}_{e, s}\right)$ may come up only when the restraint is high, so the $P$-strategy may not achieve its objective even if the lim inf of the restraint is finite. To resolve this conflict, we will, roughly speaking, make the $P$-strategy only observe (for $d_{n}^{s}$ ) the lowest restraint since some $d_{m}^{s}$ with $m \leq n$ moved. (This will be done through the control function $Q$. An alternative way to resolve this conflict would be to delay putting the elements into $A$.)

Before describing the full construction, we will define all the parameters. Let $\Lambda_{1}=\omega$ and $\Lambda_{2}=2$ be the sets of outcomes of the $\mathcal{N}$ - and $\mathcal{P}$-strategies, respectively. Let

$T=\left\{\sigma \in\left(\Lambda_{1} \cup \Lambda_{2}\right)^{<\omega} \mid(\forall i<|\sigma|)\left[\left(i\right.\right.\right.$ even $\left.\rightarrow \sigma(i) \in \Lambda_{1}\right) \wedge\left(i\right.$ odd $\left.\left.\left.\rightarrow \sigma(i) \in \Lambda_{2}\right)\right]\right\}$,

and let $T_{1}$ and $T_{2}$ be the sets of nodes of even and odd length in $T$, respectively. For each $k$, let $\left\{W_{h(k, i)}\right\}_{i \in \omega}$ be a uniformly r.e. sequence of sets such that $k \in$ $\varnothing^{(4)}$ iff $(\exists i)\left[W_{h(k, i)}\right.$ coinfinite $]$. Without loss of generality, assume that $W_{h(k, i), s} \neq$ $W_{h(k, i), s+1}$ for all $k, i, s$. The construction of $A=A_{k}$ will be controlled by markers $h_{i, s}^{n}$ where $\overline{W_{h(k, i), s}}=\left\{h_{i, s}^{0}<h_{i, s}^{1}<h_{i, s}^{2}<\cdots\right\}$.

Fix a recursive 1-1 enumeration $f$ of $V$, and let $V_{s}=\{f(0), f(1), f(2), \ldots, f(s)\}$. Let $\tilde{W}_{e, s}=\left\{x \in W_{e, s} \mid(\forall y<x)\left[y \in W_{e, s} \cup V_{s}\right]\right\}$, and let $\tilde{W}_{e}=\bigcup_{s} \tilde{W}_{e, s}$. Define the $e$-states $\sigma(e, x, s)=\left\{e^{\prime} \leq e \mid x \in \tilde{W}_{e^{\prime}, s}\right\}$, and $\sigma(e, x)=\lim _{s} \sigma(e, x, s)$. Denote the elements of the difference set $V-A$ by markers $d_{n}^{s}$ so that $V_{s}-A_{s}=$ $\left\{d_{0}^{s}, d_{1}^{s}, d_{2}^{s}, d \ldots, d_{n_{s}}^{s}\right\}$. The order of these markers will be determined by the construction, and markers $d_{n}^{s}$ will be undefined for $n>n_{s}$.

Each $\mathcal{N}$-strategy $\alpha \in T_{1}$ builds its own set $B_{\alpha}$, trying to disprove $A \subset_{\mathrm{m}} V$ by $B_{\alpha}$. It has to take into account the action of stronger $\mathcal{P}$-strategies in building $B_{\alpha}$ 
and imposing restraint of $A$. So it will use

$$
U_{\alpha, s}=\operatorname{def}\left(\left(\bigcap_{\substack{2 e^{\prime}<|\alpha| \\ \alpha\left(2 e^{\prime}+1\right)=0}} \tilde{W}_{e^{\prime}, s}\right) \cap V_{s}\right)-\left(A_{s} \cup B_{\alpha, s}\right)
$$

(instead of $V_{s}-\left(A_{s} \cup B_{\alpha, s}\right)$ as in the basic module). Notice that $U_{\alpha}={ }^{*} V-\left(A \cup B_{\alpha}\right)$ if $\alpha$ has a correct guess about the higher-priority $P$-strategies.

We define $\delta_{s}$ (with $\left|\delta_{s}\right|=2 s$ ), the recursive approximation to the true path, by induction:

(43)

$$
\begin{aligned}
\delta_{s}(2 e) & =\min \left\{n \mid h_{e, s}^{n} \neq h_{e, s^{\prime}}^{n}\right\} \text { where } s^{\prime}=\max \left(\{0\} \cup\left\{t<s \mid \delta_{s} \uparrow 2 e \subseteq \delta_{t}\right\}\right), \\
\delta_{s}(2 e+1) & =\left\{\begin{array}{c}
0 \quad \text { if } \tilde{W}_{e, s} \neq \tilde{W}_{e, s^{\prime}} \text { where } \\
s^{\prime}=\max \left(\{0\} \cup\left\{t<s \mid \delta_{s} \uparrow(2 e+1) \subseteq \delta_{t}\right\}\right), \\
1 \quad \text { otherwise. }
\end{array}\right.
\end{aligned}
$$

For $P$-strategies $\alpha=\beta^{\wedge}\langle m\rangle \in T_{2}$, define the restraint function by:

$$
r_{s}\left(\beta^{\frown}\langle m\rangle\right)=\left\{\begin{array}{l}
\min \left\{r|| U_{\alpha, s} \cap[0, r) \mid=m \vee r=1+\max \left(U_{\alpha, s}\right)\right\} \\
r_{s-1}\left(\beta^{\frown}\langle m\rangle\right) \text { otherwise. }
\end{array}\right.
$$

(Recall that restraint is imposed by $\mathcal{N}$-strategies $\beta \in T_{1}$, but the restraint that $\beta$ imposes depends on $W_{h(k, i)}$ and thus differs below distinct outcomes $m$ (the current guess for $\left.\left|\overline{W_{h(k, i)}}\right|\right)$ of $\beta$.)

For $P$-strategies $\alpha \in T_{2}$, define the control function by

$$
Q_{s}(\alpha)= \begin{cases}\infty & \text { if } \alpha \subseteq \delta_{s} \text { or } \alpha>_{L} \delta_{s} \text { or } s=0 \\ n & \text { if } \alpha<_{L} \delta_{s} \text { and } \alpha \text { moved } \Gamma_{n} \text { at stage } s \text { (as defined below) } \\ Q_{s-1}(\alpha) & \text { otherwise. }\end{cases}
$$

The construction of the r.e. set $A$ and the r.e. sets $B_{\alpha}$ (for all $\alpha \in T_{1}$ ) now proceeds as follows:

At stage 0 , let $A_{0}=B_{\alpha, 0}=\varnothing$ (for $\alpha \in T_{1}$ ), let $d_{0}^{0}=f(0)$, and let $d_{n}^{0}$ be undefined for all $n>0$.

At a stage $s+1$, perform the following two steps:

For all $\mathcal{N}$-strategies $\alpha \in T_{1}$ with $\alpha \subseteq \delta_{s}$, put $\min \left(\overline{V_{s} \cup B_{\alpha, s}}\right)$ into $B_{\alpha, s+1}$ if $\left|U_{\alpha, s}\right|>\left|B_{\alpha, s}\right|$.

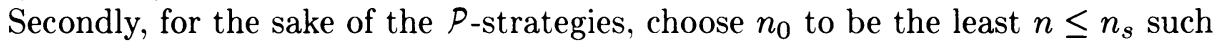
that

$$
\begin{aligned}
& (\exists e \leq n)\left[\sigma(e-1, f(s+1), s)=\sigma\left(e-1, d_{n}^{s}, s\right) \wedge\right. \\
& \begin{array}{l}
f(s+1) \in \tilde{W}_{e, s} \wedge d_{n}^{s} \notin \tilde{W}_{e, s} \wedge d_{n}^{s}>\max \left\{r_{s}(\alpha) \mid \alpha \leq \gamma \wedge \alpha \in T_{2}\right\} \\
\left.\quad\left(\text { where } \gamma \leq \delta_{s} \text { is leftmost with }|\gamma|=2 e+1 \text { and } Q_{s}(\gamma)>n\right)\right] .
\end{array}
\end{aligned}
$$

If $n_{0}$ exists then put $d_{n_{0}}^{s}$ into $A_{s+1}$, let $d_{n_{0}}^{s+1}=f(s+1)$, and let $d_{n}^{s+1}=d_{n}^{s}$ for $n \neq n_{0}$. (We say $\gamma$ moved $\Gamma_{n_{0}}$ at stage $s+1$.) Otherwise, let $d_{n_{s}+1}^{s+1}=f(s+1)$, and let $d_{n}^{s+1}=d_{n}^{s}$ for $n \neq n_{s}+1$.

This concludes the construction. 
Lemma 1 (MARKer CONVERgence Lemma). For all $n, d_{n}=\lim _{s} d_{n}^{s}$ is defined. (Thus $A \subset_{\infty} V$.)

PROOF. By induction on $n$ : Suppose $d_{m}$ is defined for all $m<n$, and $d_{m}^{s}=d_{m}$ for all $s \geq s_{0}$, say. Then $d_{n}^{s}$ is defined for all $s>s_{0}$ and changes only finitely often since it increases its $n$-state each time (and the $n$-state is nondecreasing between these changes).

Lemma 2 (True Path Existence Lemma). If $W_{h(k, i)}$ is cofinite for all $i<i_{0}$, then $\alpha_{0}=\liminf _{s} \delta_{s} \uparrow 2 i_{0}$ exists.

PROOF. By the definition of $\delta_{s}$, we have for $i<i_{0}$ :

$$
\begin{aligned}
\alpha_{0}(2 i) & =\left|\overline{W_{h(k, i)}}\right|, \\
\alpha_{0}(2 i+1) & = \begin{cases}0 & \text { if } \tilde{W}_{i} \text { is infinite } \\
1 & \text { otherwise . }\end{cases}
\end{aligned}
$$

LEMMA 3 (OUTCOME LeMMA). Fix $i_{0}$.

(i) If $\alpha_{0}=\liminf _{s} \delta_{s} \uparrow 2 i_{0}$ exists, then $\bar{V} \subseteq B_{\alpha_{0}}$, and

$$
\begin{aligned}
\beta_{0}=\alpha_{0} \hat{\langle}\langle m\rangle \wedge\left(\exists^{<\infty} s\right)\left[\delta_{s}<_{L} \beta_{0}\right] & \rightarrow \\
\left(\forall \beta \in T_{2}\right)\left[\beta \leq \beta_{0} \rightarrow r(\beta)=\right. & \left.\liminf r_{s}(\beta)<\infty \text { exists }\right] \\
& \wedge\left|U_{\alpha_{0}} \cap\left[0, r\left(\beta_{0}\right)\right)\right|=m .
\end{aligned}
$$

(ii) If $\gamma_{0}=\liminf { }_{s} \delta_{s} \uparrow\left(2 i_{0}+1\right)$ exists, then either $\tilde{W}_{i_{0}}$ is finite $\left(\right.$ if $\gamma_{0} \hat{-}\langle 1\rangle=$

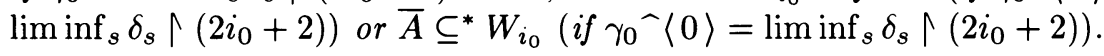

PrOOF. By simultaneous induction on $i_{0}$ :

(i) We first establish $\bar{V} \subseteq B_{\alpha_{0}}$. By the construction, it suffices to show that $B_{\alpha_{0}}$ is infinite (since we always put $\min \left(\overline{V_{s} \cup B_{\alpha_{0}, s}}\right)$ into $B_{\alpha_{0}}$ ). Suppose for the sake of a contradiction that $B_{\alpha_{0}}$ is finite. Then for all $s$ with $\alpha_{0} \subseteq \delta_{s},\left|U_{\alpha_{0}, s}\right| \leq\left|B_{\alpha_{0}, s}\right|$. But $U_{\alpha_{0}}$ is a difference of r.e. sets, so $\left|U_{\alpha_{0}}\right| \leq\left|B_{\alpha_{0}}\right|$. By (ii), $\bar{A} \subseteq \subseteq^{*} \tilde{W}_{i}$ for $i<i_{0}$ with $\alpha_{0}(2 i)=0$, and therefore $U_{\alpha_{0}}={ }^{*} V-\left(A \cup B_{\alpha_{0}}\right)$. But then $U_{\alpha_{0}}={ }^{*} V-A$ is finite, contradicting Lemma 1.

Let us now show (48). By induction on (i), choose $s_{0}$ such that

$$
\left(\forall s \geq s_{0}\right)\left(\forall \alpha \in T_{2}\right)\left[\alpha \leq \alpha_{0} \uparrow\left(2 i_{0}-1\right) \rightarrow r_{s}(\alpha)=r(\alpha)\right]
$$

(This assumption is vacuous for $i_{0}=0$.) Next, by our assumption on $\beta_{0}$ and the definition of $r_{s}(\beta)$, pick $s_{1} \geq s_{0}$ such that

$$
\left(\forall s \geq s_{1}\right)\left(\forall \beta \in T_{2}\right)\left[\beta<\beta_{0} \wedge \beta \uparrow(|\beta|-1) \neq \alpha_{0} \rightarrow r_{s}(\beta)=r(\beta)\right] .
$$

Furthermore, since by the construction $Q_{s}(\beta)$ cannot increase while $\beta<_{L} \delta_{s}$, and since $\beta$ has a correct guess about the $P_{\text {-strategies }} \gamma \subset \beta$, pick $s_{2} \geq s_{1}$ such that

$$
\begin{aligned}
&\left(\forall s \geq s_{2}\right)\left(\forall \beta \in T_{2}\right)[\left(\beta<_{L} \beta_{0} \rightarrow Q_{s}(\beta)=\lim _{t} Q_{t}(\beta)\right) \wedge \\
&\left(\forall \gamma \subset \beta_{0}\right)\left[|\gamma|=2 i+1 \wedge \beta_{0}(2 i+1)=1 \rightarrow \gamma\right. \\
&\text { does not move any element at stage } s]] .
\end{aligned}
$$

Finally, let $\sigma=\left\{i<i_{0} \mid \tilde{W}_{i}\right.$ infinite $\}$. Then by (ii),

$$
\left(\exists n_{0}\right)\left(\forall n \geq n_{0}\right)\left[\sigma\left(i_{0}-1, d_{n}\right)=\sigma\right] .
$$


Pick $s_{3} \geq s_{2}$ such that

$$
\left(\forall s \geq s_{3}\right)\left(\forall n<n_{0}\right)\left[d_{n}^{s}=d_{n}\right]
$$

We will now show (48) by induction on $m$ (for fixed $\alpha_{0}$ ). For $m=0$, trivially $r\left(\beta_{0}\right)=0$. Let $m>0$. Let $r=1+\max \left(\left\{r\left(\alpha_{0} \widehat{\langle}\langle m-1\rangle\right)\right\} \cup\left\{d_{n} \mid n<n_{0}\right\}\right)$. Pick $s_{4} \geq s_{3}$ such that

$$
\begin{aligned}
& \left(\forall s \geq s_{4}\right)\left[r_{s}\left(\alpha_{0} \widehat{\langle}\langle m-1\rangle\right)=r\left(\alpha_{0} \widehat{\langle}\langle m-1\rangle\right) \wedge\right. \\
& \left.\left.X_{s_{4}} \uparrow(r+1)=X \uparrow(r+1) \text { for all } X=W_{i} \text { (for } i<i_{0}\right), V, A \text {, and } B_{\alpha_{0}}\right] \text {. }
\end{aligned}
$$

By the first part of (i), we have $\lim \sup \left\{\left|U_{\alpha_{0}, s}\right| \mid \alpha_{0} \leq \delta_{s}\right\}=\infty$, so pick $s_{5} \geq s_{4}$ such that $\alpha_{0} \subseteq \delta_{s_{5}}$ and $\left|U_{\alpha_{0}, s_{5}}\right| \geq m$.

We claim that

$$
\left(\forall s \geq s_{5}\right)\left[r_{s}\left(\beta_{0}\right) \geq r_{s+1}\left(\beta_{0}\right) \wedge\left|U_{\alpha_{0}, s} \cap\left[0, r_{s}\left(\beta_{0}\right)\right)\right| \geq m\right] .
$$

Suppose for the sake of a contradiction that for some $s \geq s_{5}, U_{\alpha_{0}, s} \cap\left[0, r_{s}\left(\beta_{0}\right)\right) \nsubseteq$ $U_{\alpha_{0}, s+1} \cap\left[0, r_{s}\left(\beta_{0}\right)\right)$. Then some $x \in U_{\alpha_{0}, s}$ entered $B_{\alpha_{0}}$ or $A$. The former is impossible by the construction of $B_{\alpha_{0}}$ (since $x \in V_{s}$ ). But $x$ cannot enter $A$ since:

(a) no $\gamma \geq \beta_{0} \widehat{\langle}\langle m\rangle$ can move $x$ by the restraint imposed;

(b) no $\gamma<_{L} \beta_{0} \widehat{\langle}\langle m\rangle$ can move $x$, or else $Q_{s}(\gamma)>Q_{s+1}(\gamma)$, contradicting the assumption on $s_{2}$; and

(c) no $\gamma \subset \beta_{0}$ will move $x$ since either $x \notin \tilde{W}_{i, s}$ (if $|\gamma|=2 i+1$ and $\beta_{0}(2 i+1)=$ 0 ), or $\gamma$ no longer moves any element (if $|\gamma|=2 i+1$ and $\beta_{0}(2 i+1)=1$ ).

(Notice that $r_{s}\left(\beta_{0}\right)$ may still drop a finite number of times as $U_{\alpha_{0}}$ gets new small elements.)

Now (55) establishes (48).

(ii) By (i), pick $s_{0}$ such that

$$
\left(\forall s \geq s_{0}\right)\left(\forall \gamma \in T_{2}\right)\left[\gamma \leq \gamma_{0} \rightarrow r(\gamma)=r_{s}(\gamma)\right] .
$$

Let $R\left(\gamma_{0}\right)=\max \left\{r(\gamma) \mid \gamma \leq \gamma_{0} \wedge \gamma \in T_{2}\right\}$. Since $\gamma_{0} \subseteq \delta_{s}$ for infinitely many $s$, we also have $\lim _{s} Q_{s}\left(\gamma_{0}\right)=\infty$. Let $\sigma=\left\{i \leq i_{0} \mid \tilde{W}_{i}\right.$ infinite $\}$, and assume that $\tilde{W}_{i_{0}}$ is infinite. Then $\tilde{W}_{\sigma}=\bigcap_{i \in \sigma} \tilde{W}_{i} \supseteq \bar{V}$. By induction on (ii), pick $n_{0}>i_{0}$ such that

$$
\left(\forall n \geq n_{0}\right)\left[\sigma\left(i_{0}-1, d_{n}\right)=\sigma-\left\{i_{0}\right\}\right]
$$

Since $V$ is not recursive,

$$
\left(\exists^{\infty} s\right)\left[f(s+1) \in \tilde{W}_{\sigma, s}\right] .
$$

Suppose that $\sigma\left(i_{0}, d_{n}\right)=\sigma-\left\{i_{0}\right\}$ for some $n \geq n_{0}$ with $d_{n}>R\left(\gamma_{0}\right)$. Pick $s_{1} \geq s_{0}$ such that

$$
\left(\forall s \geq s_{1}\right)\left[Q_{s}\left(\gamma_{0}\right)>n \wedge\left(\forall n^{\prime} \leq n\right)\left[d_{n^{\prime}}^{s}=d_{n^{\prime}}\right]\right] .
$$

Then $d_{n}$ will be moved by (58), contradicting our assumption. Thus $W_{i_{0}}=\tilde{W}_{i_{0}} \supseteq^{*}$ $\bar{A}$.

It is now easy to see that the lemmas imply Theorem D.

First suppose that $k \in \varnothing^{(4)}$. Then $W_{h\left(k, i_{0}\right)}$ is coinfinite for some (least) $i_{0}$. By Lemma $2, \alpha_{0}=\liminf _{s} \delta_{s} \uparrow 2 i_{0}$ exists, and

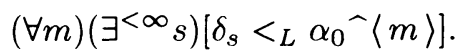


Therefore, by Lemma 3 (i), $\bar{V} \subseteq B_{\alpha_{0}}$, and $U_{\alpha_{0}}$ is infinite. But then $V-\left(A \cup B_{\alpha_{0}}\right)$ is infinite, so $B_{\alpha_{0}}$ witnesses that $A \not \subset_{\mathrm{m}} V$.

On the other hand, assume that $k \notin \varnothing^{(4)}$. Then $W_{h(k, i)}$ is cofinite for all $i$. By Lemma $2, \liminf \delta_{s} \delta_{s} \uparrow 2 i$ exists for all $i$. Therefore, by Lemma 3 (ii), either $\tilde{W}_{i}$ is finite or $\bar{A} \subseteq{ }^{*} \tilde{W}_{i}=W_{i}$ for all $i$. Furthermore, by Lemma $1, A \subset_{\infty} V$. Thus $A \subset \mathrm{m} V$.

This concludes the proof of Theorem D.

\section{REFERENCES}

[Fr58] R. M. Friedberg, Three theorems on recursive enumeration: I. Decomposition, II. Maximal set, III. Enumeration without duplication, J. Symbolic Logic 23 (1958), 309-316.

[Je78] T. Jech, Set theory, Academic Press, New York, 1978.

[JLSSta] C. G. Jockusch, Jr., M. Lerman, R. I. Soare and R. M. Solovay, Recursively enumerable sets modulo iterated jumps and extensions of Arslanov's completeness criterion, in preparation.

[La68] A. H. Lachlan, On the lattice of recursively enumerable sets, Trans. Amer. Math. Soc. 130 (1968), 1-37.

[Ro67] H. Rogers, Jr., Theory of recursive functions and effective computability, McGrawHill, New York, 1967.

[Sota] R. I. Soare, Recursively enumerable sets and degrees, Springer-Verlag, Heidelberg, 1987.

[Tu71] R. E. Tulloss, Some complexities of simplicity concerning grades of simplicity of recursively enumerable sets, Ph.D. Dissertation, Univ. of California, Berkeley, 1971.

Department of Mathematics, Yale University, New haven, Connecticut 06520 